\title{
THE APOKASC CATALOG: AN ASTEROSEISMIC AND SPECTROSCOPIC JOINT SURVEY OF TARGETS IN THE KEPLER FIELDS
}

Marc H. Pinsonneault ${ }^{1,2}$, Yvonne Elsworth ${ }^{3,4}$, Courtney Epstein $^{1}$, Saskia Hekker ${ }^{4,5}$, Sz. Mészáros $^{6,7}$,

William J. Chaplin ${ }^{3,4}$, Jennifer A. Johnson ${ }^{1,2}$, Rafael A. García ${ }^{8}$, Jon Holtzman ${ }^{9}$, Savita Mathur ${ }^{10}$, Ana García Pérez ${ }^{11}$, Victor Silva Aguirre ${ }^{4}$, Léo Girardi ${ }^{12,13}$, Sarbani Basu ${ }^{14}$, Matthew Shetrone ${ }^{15}$, Dennis Stello ${ }^{4,16}$, Carlos Allende Prieto ${ }^{17,18}$, Deokkeun An ${ }^{19}$, Paul Beck ${ }^{8}$, Timothy C. Beers ${ }^{20,21}$, Dmitry Bizyaev ${ }^{22}$, Steven Bloemen ${ }^{23}$, Jo Bovy ${ }^{24}$, Katia Cunha ${ }^{25,26}$, Joris De Ridder ${ }^{27}$, Peter M. Frinchaboy ${ }^{28}$, D. A. García-Hernández ${ }^{17,18}$,

Ronald Gilliland ${ }^{29}$, Paul Harding ${ }^{30}$, Fred R. Hearty ${ }^{29}$, Daniel Huber ${ }^{31,32}$, Inese Ivans $^{33}$, Thomas Kallinger ${ }^{34}$, Steven R. Majewski ${ }^{11}$, Travis S. Metcalfe ${ }^{10}$, Andrea Miglio ${ }^{3,4}$, Benoit Mosser $^{35}$, Demitri Muna ${ }^{1}$, David L. Nidever ${ }^{36}$, Donald P. Schneider ${ }^{29,37}$, Aldo Serenelli ${ }^{38}$, Verne V. Smith ${ }^{39}$, Jamie Tayar ${ }^{1}$, Olga Zamora ${ }^{17,18}$, And Gail Zasowski ${ }^{40}$

${ }^{1}$ Department of Astronomy, The Ohio State University, Columbus, OH 43210, USA; pinsonneault.1@osu.edu

${ }^{2}$ Center for Cosmology and Astroparticle Physics, The Ohio State University, Columbus, OH 43210, USA

${ }^{3}$ University of Birmingham, School of Physics and Astronomy, Edgbaston, Birmingham B15 2TT, UK

${ }^{4}$ Stellar Astrophysics Centre, Department of Physics and Astronomy, Aarhus University, Ny Munkegade 120, DK-8000 Aarhus C, Denmark

${ }^{5}$ Max-Planck-Institut für Sonnensystemforschung, Justus-von-Liebig-Weg 3, D-37077 Gottingen, Germany

${ }^{6}$ Astronomy Department, Indiana University, Bloomington, IN 47405, USA

${ }^{7}$ ELTE Gothard Astrophysical Observatory, H-9704 Szombathely, Szent Imre herceg st, 112, Hungary

${ }^{8}$ Laboratoire AIM, CEA/DSM-CNRS-Université Denis Diderot-IRFU/SAp, F-91191 Gif-sur-Yvette Cedex, France

${ }^{9}$ Department of Astronomy, MSC 4500, New Mexico State University, P.O. Box 30001, Las Cruces, NM 88003, USA

${ }^{10}$ Space Science Institute, 4750 Walnut street, Suite 205, Boulder, CO 80301, USA

${ }^{11}$ Department of Astronomy, University of Virginia, P.O. Box 400325, Charlottesville, VA 22904-4325, USA

${ }^{12}$ Osservatorio Astronomico di Padova-INAF, Vicolo dell'Osservatorio 5, I-35122 Padova, Italy

${ }^{13}$ Laboratório Interinstitucional de e-Astronomia-LIneA, Rua Gal. José Cristino 77, Rio de Janeiro, RJ 20921-400, Brazil

${ }^{14}$ Department of Astronomy, Yale University, P.O. Box 208101, New Haven, CT 06520-8101, USA

${ }^{15}$ University of Texas at Austin, McDonald Observatory, 32 Fowlkes Road, TX 79734-3005, USA

${ }^{16}$ Sydney Institute for Astronomy (SIfA), School of Physics, University of Sydney, NSW 2006, Australia

${ }^{17}$ Instituto de Astrofsica de Canarias (IAC), C/Va Lactea, s/n, E-38200 La Laguna, Tenerife, Spain

${ }^{18}$ Departamento de Astrofsica, Universidad de La Laguna, E-38206 La Laguna, Tenerife, Spain

${ }^{19}$ Department of Science Education, Ewha Womans University, Seoul, Korea

${ }^{20}$ Department of Physics, University of Notre Dame, 225 Nieuwland Science Hall, Notre Dame, IN 46656, USA

${ }^{21}$ JINA - Joint Institute for Nuclear Astrophysics, University of Notre Dame, Notre Dame, IN 46556, USA

${ }^{22}$ Apache Point Observatory and New Mexico State University, P.O. Box 59, Sunspot, NM 88349-0059, USA

${ }^{23}$ Department of Astrophysics, IMAPP, Radboud University Nijmegen, P.O. Box 9010, NL-6500 GL Nijmegen, The Netherlands

${ }^{24}$ Institute for Advanced Study, Einstein Drive, Princeton, NJ 08540, USA

${ }^{25}$ Observatório Nacional, Sao Cristóvão, Rio de Janeiro, Brazil

${ }^{26}$ Steward Observatory, University of Arizona, Tucson, AZ 85719, USA

${ }^{27}$ Instituut voor Sterrenkunde, KU Leuven, B-3001, Leuven, Belgium

${ }^{28}$ Department of Physics and Astronomy, Texas Christian University, Fort Worth, TX 76129, USA

${ }^{29}$ Department of Astronomy and Astrophysics, The Pennsylvania State University, University Park, PA 16802, USA

${ }^{30}$ Department of Astronomy, Case Western Reserve University, Cleveland, OH 44106-7215, USA

${ }^{31}$ NASA Ames Research Center, Moffett Field, CA 94035, USA

${ }^{32}$ SETI Institute, 189 Bernardo Avenue, Mountain View, CA 94043, USA

${ }^{33}$ Department of Physics and Astronomy, The University of Utah, Salt Lake City, UT 84112, USA

${ }^{34}$ Institute for Astronomy, University of Vienna, Türkenschanzstrasse 17, A-1180 Vienna, Austria

${ }^{35}$ LESIA, UMR 8109, Université Pierre et Marie Curie, Université Denis Diderot, Observatoire de Paris, F-92195 Meudon Cedex, France

36 Department of Astronomy, University of Michigan, Ann Arbor, MI 48104, USA

${ }^{37}$ Institute for Gravitation and the Cosmos, The Pennsylvania State University, University Park, PA 16802, USA

${ }^{38}$ Institute of Space Sciences (IEEC-CSIC), Campus UAB, E-08193 Bellaterra, Spain

${ }^{39}$ National Optical Astronomy Observatories, Tucson, AZ 85719, USA

${ }^{40}$ Department of Physics and Astronomy, Johns Hopkins University, Baltimore, MD 21218, USA

Received 2014 June 27; accepted 2014 October 3; published 2014 December 2

\begin{abstract}
We present the first APOKASC catalog of spectroscopic and asteroseismic properties of 1916 red giants observed in the Kepler fields. The spectroscopic parameters provided from the Apache Point Observatory Galactic Evolution Experiment project are complemented with asteroseismic surface gravities, masses, radii, and mean densities determined by members of the Kepler Asteroseismology Science Consortium. We assess both random and systematic sources of error and include a discussion of sample selection for giants in the Kepler fields. Total uncertainties in the main catalog properties are of the order of $80 \mathrm{~K}$ in $T_{\text {eff }}, 0.06$ dex in $[\mathrm{M} / \mathrm{H}], 0.014$ dex in $\log g$, and $12 \%$ and $5 \%$ in mass and radius, respectively; these reflect a combination of systematic and random errors. Asteroseismic surface gravities are substantially more precise and accurate than spectroscopic ones, and we find good agreement between their mean values and the calibrated spectroscopic surface gravities. There are, however, systematic underlying trends with $T_{\text {eff }}$ and $\log g$. Our effective temperature scale is between 0 and $200 \mathrm{~K}$ cooler than that expected from the infrared flux method, depending on the adopted extinction map, which provides evidence for a lower value on average than that inferred for the Kepler Input Catalog (KIC). We find a reasonable correspondence between the photometric KIC and spectroscopic APOKASC metallicity scales, with increased dispersion in KIC metallicities as
\end{abstract}


the absolute metal abundance decreases, and offsets in $T_{\text {eff }}$ and $\log g$ consistent with those derived in the literature. We present mean fitting relations between APOKASC and KIC observables and discuss future prospects, strengths, and limitations of the catalog data.

Key words: catalogs - stars: abundances - stars: fundamental parameters - stars: oscillations (including pulsations) - surveys

Online-only material: color figures, machine-readable tables

\section{INTRODUCTION}

We are entering an era of precision stellar astrophysics. Large surveys are yielding data with unprecedented quality and quantity, and even more ambitious programs are on the near horizon. This advance is not merely a matter of much larger samples of measurements than were possible before; there are also fundamentally new observables arising from the advent of asteroseismology as a practical stellar population tool. These new observables are particularly powerful diagnostics when complemented with data from more traditional approaches. In this paper we present the first release of the joint APOKASC asteroseismic and spectroscopic survey for targets with both high-resolution Apache Point Observatory Galactic Evolution Experiment (APOGEE) spectra analyzed by members of the third Sloan Digital Sky Survey (SDSS-III) and asteroseismic data obtained by the Kepler mission and analyzed by members of the Kepler Asteroseismology Science Consortium (KASC). When completed, we anticipate of the order of 8000 red giants and 600 dwarfs and subgiants with asteroseismic data and highresolution spectra. In this initial paper, we catalog the properties of 1916 red giants observed as part of the SDSS DR10 (Ahn et al. 2014). A catalog for the less-evolved stars will be presented in a separate publication (A. Serenelli et al. 2014, in preparation).

Large spectroscopic surveys in the Milky Way are now a reality, and a variety of sampling strategies and resolutions have been employed. Low- to medium-resolution surveys such as SEGUE (Yanny et al. 2009), RAVE (Kordopatis et al. 2013), and LAMOST (Zhao et al. 2006) provide stellar properties for large samples of stars. High-resolution programs are complementary, with more detailed abundance mixtures and more precise measurements for still substantial samples. GALAH (Freeman 2012) and Gaia-ESO (Gilmore et al. 2012) are optical surveys; APOGEE (Majewski et al. 2010; Hayden et al. 2014) is instead focusing on the infrared (IR). Infrared spectroscopy is attractive for Milky Way studies because it is less sensitive to extinction; it also has different systematic error sources than traditional optical spectroscopy (A. García Pérez 2014, in preparation). These surveys permit detailed stellar population reconstructions using chemical tagging, kinematic data, effective temperature, and surface gravities; see, for example, Bovy et al. (2012) for SEGUE, Bergemann et al. (2014) for Gaia-ESO, or Binney et al. (2014) for RAVE. Spectroscopic properties can be complemented by photometric parameter estimation, and the Gaia mission (Perryman et al. 2001) should add critical measurements of distances and proper motions.

Standing alone, however, there are intrinsic limitations in the information from spectroscopic studies of stellar populations. The fundamental stellar properties of mass and age are only indirectly inferred from spectra. In the case of red giants, their H-R diagram position yields relatively weak constraints on either mass or age. Chemical tagging-for example, using high $[\alpha / \mathrm{Fe}]$ as a marker of old populations (Wallerstein 1962; Tinsley 1979) — is a valuable method, but the absolute timescale is uncertain (Matteucci et al. 2009) and the chemical evolution rates in different systems need not have been the same. Large surveys also require automated pipeline estimation of stellar parameters, and it can be difficult to calibrate these pipelines. These issues reflect the underlying problem that the dependence of absorption line strength on stellar atmosphere properties is not completely understood. This is in part due to incomplete and inaccurate atomic data, but there are also important physical effects that are challenging to model. Traditional atmosphere analysis uses one-dimensional plane-parallel atmospheres, treats line broadening with an ad hoc microturbulence, and assumes local thermodynamic equilibrium (LTE). Spherical effects, more realistic turbulence modeling from threedimensional hydrodynamic simulations, and departures from LTE can strongly impact the interpretation of the spectrum (see, for example, Asplund 2005). The difficulty of modeling the outermost layers, where real atmospheres transition to a chromosphere, can also play a role in complicating spectroscopic inferences. Therefore, stellar parameter determinations for large samples require calibration against standards whose properties are known by other means, or whose membership in a cluster demands that they have similar composition.

Independently, there have been exciting advances in asteroseismology driven largely by data from space missions. In an important breakthrough, the CoRoT (De Ridder et al. 2009) and Kepler (Bedding et al. 2010) missions have discovered that virtually all red giants are non-radial oscillators. Red giant asteroseismology is revolutionizing our understanding of stellar structure and evolution. Global properties of the oscillations, such as the large frequency spacing $\Delta v$ and frequency of maximum oscillation power $v_{\max }$, are naturally related to the stellar mean density and surface gravity, respectively (Kjeldsen \& Bedding 1995); see also Stello et al. (2009a) and Huber et al. (2011). Basic pulsation data, combined with measured effective temperatures, permit estimation of stellar masses and radii in a domain where these crucial stellar properties have been notoriously uncertain. Access to large numbers of stellar mass measurements has profound implications for stellar population studies (Miglio et al. 2009; Freeman 2011). Seismology also yields completely new observables for red giants because of a fortunate coincidence: their physical structure permits coupling between waves that propagate primarily in the core and those that propagate primarily in the envelope. The net result is oscillations of mixed character that carry information about the structure of both the core and envelope. We can therefore use the detailed pattern of observed oscillation frequencies to distinguish between first-ascent giants (with $\mathrm{H}$-shell burning only) and red-clump (RC) stars (with He-core and H-shell burning) (Bedding et al. 2011). Asymptotic red giant stars, with two shell sources, would appear with a pattern similar, but not identical, to first-ascent giants; however, most work to date has focused on lower luminosities where such stars are not expected to be found. Rapid rotation in the cores of red giants has been discovered (Beck et al. 2012; Deheuvels et al. 2012; Mosser et al. 
2012a). Time series data from Kepler can also measure the surface rotation rates of stars through starspot modulation of their light curves (Basri et al. 2011; García et al. 2014b; McQuillan et al. 2014). Mapping the angular momentum evolution of giants as a function of mass is another new frontier with rich astrophysical reward.

Asteroseismology alone, however, has important limitations on the information that it can provide. Effective temperatures are required to infer mass and radius separately, and stellar ages depend on both mass and composition. For example, it was possible to use the Kepler Input Catalog (KIC) data of Brown et al. (2011) to define a sequence of solar-mass asteroseismic targets from the main sequence to the giant branch, but abundances are essential for finding true solar analogs (Silva Aguirre et al. 2011). In the CoRoT fields Miglio et al. (2013) were able to infer mean stellar mass differences between populations along different sightlines, but interpreting these measurements in terms of age would require spectroscopic data on metallicity and the mixture of heavy elements. The scarcity of abundance data for stars in the Kepler field is therefore a major limitation; the sheer volume of data (roughly 21,000 red giants) has made traditional spectroscopic studies infeasible.

Fortunately, there is a new spectroscopic survey ideally suited for large samples of red giants. The multi-fiber, highresolution $H$-band spectrograph from APOGEE on the SDSS $2.5 \mathrm{~m}$ telescope (Gunn et al. 2006) is ideally suited to observing Kepler targets because it is well matched to the target density of the fields observed by the mission with 230 science fibers available over a $7 \mathrm{deg}^{2}$ field. The $R=22,500$ spectra were designed to produce temperatures, $[\mathrm{Fe} / \mathrm{H}]$, and $[\mathrm{X} / \mathrm{Fe}]$ with accuracies of $4 \%, 0.1 \mathrm{dex}$, and $0.1 \mathrm{dex}$, respectively (Eisenstein et al. 2011). Actual performance, as reported by Meszaros et al. (2013), is close to these goals. There is no other spectroscopic sample of this size and quality available for Kepler red giants. ${ }^{41}$

Here we report the first APOKASC data release, which includes red giants whose spectra were released in the SDSS-III Data Release 10 (DR10), as described in Ahn et al. (2014). Our paper is organized as follows: we describe our sample selection in Section 2. Our spectroscopic data calibration is described and compared with photometric temperatures and asteroseismic surface gravities in Section 3. The asteroseismic analysis is discussed in Section 4. We present the catalog and compare it to the KIC in Section 5. A look ahead to the full catalog and a discussion is presented in Section 6.

\section{SAMPLE SELECTION}

Our goal is to obtain data for a combined asteroseismic and spectroscopic sample of a large number of astrophysically interesting targets in the Kepler fields. Understanding the selection effects in our sample is important for interpreting our results. Selection effects in our sample enter at several distinct levels: the match between the oscillation frequencies and the time sampling, the selection of which stars to study for oscillations, and the selection of targets for spectroscopic observation.

The oscillation frequencies span a broad range, from five minutes for the Sun to tens of days for luminous giants. A single observing strategy will therefore not work across the entire

\footnotetext{
41 The APOGEE data used in this paper adopted for an abundance scale the metallicity index $[\mathrm{A} / \mathrm{H}]$, which corresponds to the metallicity $[\mathrm{Fe} / \mathrm{H}]($ see Section 3.1.) Future APOGEE data releases will provide abundance measurements of 15 elements, including $\mathrm{O}, \mathrm{Mg}$, and $\mathrm{Fe}$.
}

domain. Fortunately, Kepler has two observing modes: 1 minute (short cadence) and 30 minutes (long cadence). Long-cadence targets are typically observed in 90 day cycles, hereafter referred to as quarters. The short-cadence mode is ideal for asteroseismic studies of dwarfs and subgiants; however, there are a limited number of such targets that Kepler was able to observe. Chaplin et al. (2011) detected oscillations in $\sim 600$ of the $\sim 2000$ targets observed in short-cadence mode for at least 30 days. The longcadence mode is ideal for measuring oscillations in red giants, and a large number were observed for at least one quarter by the satellite. More than $70 \%$ of the long-cadence red giants were detected as solar-like oscillators even with the first three months of data (Hekker et al. 2011b), with the non-detections primarily in luminous stars requiring a longer time sequence. Furthermore, the most precise measurements derived from IR spectra are those for cool and evolved stars, so there is a natural pairing between APOGEE spectra and asteroseismology of red giants. The bulk of our sample is therefore composed of red giants, with smaller, separate designated dwarf and subgiant cohorts, and the duration of the mission and time sampling should not introduce significant biases in our sample.

However, there are more bright red giants in the Kepler fields than were observed in long-cadence mode, and there are more long-cadence targets than the number for which it was practical to obtain spectra. There are therefore two distinct selection criteria important for this sample: the criteria for being observed by Kepler and the criteria for being observed spectroscopically by APOGEE. Furthermore, the initial DR10 sample is a subset of the overall APOKASC sample, and the properties of these fields need not be representative for the sample as a whole. We therefore begin with a summary of the Kepler short- and longcadence target selection procedures. We then describe how we used preliminary asteroseismic data to identify populations for spectroscopic observation. We then discuss special populations which we targeted for observing, describe our main sample grid, and how we filled the remaining fibers for the campaign. We end with a brief discussion of the properties of the first data set being released in this paper.

\subsection{Kepler Target Selection}

We included 400 of the asteroseismically detected subgiants and dwarfs reported in Chaplin et al. (2011) from short-cadence observations. This sample is smaller than that of Chaplin et al. (2011) because hotter dwarfs did not fit our global criteria for APOGEE observations. The selection process of targets from the long-cadence sample is more complex than that for the shortcadence sample. The Kepler mission was designed to search for transits of host stars by extrasolar planets, with a focus on solar analogs. However, giants are much more numerous than dwarfs in a sample with the Kepler magnitude limit, which was designed for stars brighter than Kepler magnitude $K_{p}=$ 16. The KIC (Brown et al. 2011) was therefore constructed to separate dwarfs and giants and to define the planet candidate target list. There were multiple criteria used to select giants for long-cadence observations. For our purposes there are two important samples: the KASC giants, defined below, and the full sample (hereafter referred to as the public giants).

A pre-launch list of 1006 targets was chosen using only information available from the KIC, and with the express purpose of providing a uniformly spaced set of stars over the focal plane serving as low proper motion, small parallax, astrometric controls. As such they were selected on the basis of a metric that was a combination of (1) large distance, 
(2) bright, but not expected to saturate the detector to allow precise centroiding, (3) uncrowded-also to support precise centroiding, and (4) spread over the focal plane to give 11-12 red giant controls for each of Kepler's 84 channels. There was also a sample of $\sim 800$ giants for asteroseismic monitoring that was assembled from pre-launch proposals submitted by KASC working groups. ${ }^{42}$ The initial red giant asteroseismology results were based on the combination of these two data sets; hereafter, we refer to these targets as the KASC giants. Huber et al. (2010) described how the basic seismic parameters were measured; Kallinger et al. (2010) provided the stellar parameters, such as mass and radius; and Hekker et al. (2011a) compared different analysis techniques. However, the derived stellar parameters did not include high-resolution spectroscopic metallicities and effective temperature estimates independent of the KIC, which APOGEE can now provide.

Characterizing the full public red giant sample is surprisingly challenging. The target list varied from observing quarter to quarter, as the giants candidates were deprioritized relative to the dwarfs for planet searches. Using data from the first 16 quarters in the Q1-Q16 star properties catalog, Huber et al. (2014) combined the KIC and published literature information to obtain a total of 21,427 stars with $\log g<3.5$ and $T_{\text {eff }}<5500$ $\mathrm{K}$ that were observed for at least one quarter during the Kepler mission. This sample does not include stars observed during commissioning (Q0) only.

The majority of the Huber et al. (2014) sample, of the order of 15,000 red giants, were selected as planet search candidates using the procedure described in Batalha et al. (2010). A total of 5282 red giants brighter than $K_{p}=14$ were included in the highest-priority planet search cohort of 150,000 stars. The remaining red giants were selected from a much larger secondary target list of 57,010 giants, including a large number $(11,057)$ brighter than $K_{p}=14$. In practice this criterion favored brighter targets that were classified as smaller red giants; this is roughly equivalent to a sample with magnitude and surface gravity cuts. The mission also supplemented the target list with a cohort of 12,000 brighter targets, including stars without KIC classification; $\sim 3300$ of these unclassified stars proved to be cool or luminous giants (Huber et al. 2014). Approximately 1000 giant targets were also added in the GO program, including 300 that were observed in quarters $14-16$ as part of a dedicated APOKASC GO proposal No. 40033 as described below. There are also an unknown number of stars classified as dwarfs in the KIC that could be giants, and vice versa. An asteroseismic luminosity classification of all cool dwarfs is planned and should yield a complete census of giant stars in 2014 .

\subsection{Target Selection for the Overall APOKASC Sample}

Observing the entire Kepler red giant sample with APOGEE was not feasible, so APOKASC had to develop an independent spectroscopic target selection process. There are high-priority categories of targets where we attempted to be as complete as possible-for example, rare but astrophysically interesting metal-poor stars or open cluster members. We also wanted uniform spectroscopic data for the stars with the highestquality Kepler light curves, as these could serve as precise calibrators for stellar population and asteroseismic studies. Another survey goal was to extend the range of surface gravity and metallicity relative to prior spectroscopic studies. Finally, ages can be inferred from masses for first-ascent red giants,

\footnotetext{
$\overline{42}$ A full list of proposals can be accessed on the KASOC database.
}

so it was important to have estimates of evolutionary state to preferentially target such stars over the more common core-He burning stars where mass loss complicates the mapping from mass to age.

Our procedures for creating the final target list is described below. For a further discussion of APOGEE and APOKASC targeting, see Zasowski et al. (2013). For all targets we adopted limits on the magnitude $(7<H<11)$ and effective temperature $\left(T_{\text {eff }}<6500 \mathrm{~K}\right)$ that were necessary for APOGEE, and we required that the targets fit within the APOGEE field of view. The magnitude limits guarded against overexposure and ensured a high signal-to-noise ratio in $1 \mathrm{hr}$ observations; the temperature cut was designed to avoid hot stars with uninformative IR spectra. We performed a uniform analysis of the Kepler light curves to check for evolutionary diagnostics, rotation, or unusual asteroseismic properties. We added in external information for interesting populations. We then defined a reference set of red giants and RC stars sampling a wide range of surface gravities, prioritizing stars with more complete time coverage. These data sets left us with free fibers that we could fill from the remainder of the available giant sample.

\subsubsection{Asteroseismic Classification}

We employed asteroseismic diagnostics for the full Kepler red giant sample observed in long-cadence mode to infer their evolutionary state and to identify stars with unusual pulsational properties. We discuss the methods used for extracting the basic asteroseismic observables for the catalog in Section 4. Our automated methodology for evolutionary state classification is described in Stello et al. (2013) and summarized here. The key tasks are identifying the frequencies of the dipole (angular degree $l=1$ ) oscillation modes and inferring whether the pattern is characteristic of a core He-burning star or one with a degenerate and inert $\mathrm{He}$ core. To obtain the frequencies we detrended the time series from the public Q1-Q8 data by removing discontinuities and applying a high-pass filter. The SYD pipeline (Huber et al. 2009) was used to derive the large frequency separation, and we implemented a simple peak bagging approach using different degrees of smoothing to identify all significant peaks in the power spectra. We associate a degree $l$ to each extracted frequency based on a method similar to that provided by Mosser et al. (2011b). For the purpose of measuring period spacings of the dipole modes, which is important for this classification step, we remove the radial and quadrupole modes.

We only kept stars for which we detected at least five $l=1$ modes in order to obtain a more robust result in the following step. There are more than 8000 stars that pass this criterion. For each star we measure the pairwise period spacing, $\Delta P$, between successive peaks, and take the median of $\Delta P$ as the representative period spacing (the median proved to be the most robust quantity compared to a simple mean or the moment). This method produced our best diagnostic of evolutionary state. We complemented this approach with a double-check using the methodology of Mosser et al. (2011a). A subset of our targets (3128) could be unambiguously assigned to either the $\mathrm{RC}$ or the first-ascent red giant branch (RGB). Limited frequency resolution and other backgrounds, such as rotation, made automated classification ambiguous for the remainder of the sample. This evolutionary state information was used only for target selection purposes, as our procedure was not designed to provide complete information for the entire sample. 


\subsubsection{KASC Giants, Open Cluster Members, Asteroseismic Dwarfs, and Subgiants}

We began our program by defining the highest-priority targets. The KASC giants described above are the best studied stars with the highest-quality data sets, so we ensured that all of them would be included as targets. All subgiants and dwarfs from Chaplin et al. (2011) with KIC $T_{\text {eff }}<6500 \mathrm{~K}$ and asteroseismic detections were targeted. Known members of the open clusters NGC 6811, 6819, and 6791 bright enough for APOGEE and with asteroseismic detections were also all included (Stello et al. 2011b). We also developed criteria to preferentially select metal-poor giants, rapid rotators, and luminous giants for spectroscopic observation.

\subsubsection{Metal-poor Giants}

Observing oscillations in metal-poor giants is critical for both stellar physics (e.g., scaling relations as a function of metallicity; see Epstein et al. 2014) and stellar populations questions, such as the age of the halo. A simulation of the stellar populations in the Kepler fields with the TRILEGAL code (Girardi et al. 2005) indicated that only $0.6 \%$ of giants are expected to have $[\mathrm{Fe} / \mathrm{H}]<-2.0$, for a predicted total of $\sim 100$ in the entire public giant database. Efficient targeting of these stars is therefore essential, and we employed several methods to identify candidates. A total of 23 targets were selected by having kinematics consistent with the halo, defined here as follows: a proper motion greater than $0^{\prime} .01 \mathrm{yr}^{-1}$ and a transverse velocity greater than $200 \mathrm{~km} \mathrm{~s}^{-1}$ (Brown et al. 2011). An additional 41 targets were chosen from low-resolution spectra obtained by the SDSS-III collaboration for MARVELS target pre-selection in the Kepler field. These spectra are similar to the spectra for the SEGUE and SEGUE2 surveys and were processed by the SEGUE stellar parameter pipeline, which has been shown to measure $[\mathrm{Fe} / \mathrm{H}]$ with an uncertainty of 0.25 dex (Lee et al. 2008) and to successfully identify even metal-poor giants (Lai et al. 2009), which are challenging to study at low resolution. Finally, 67 metal-poor candidates were selected on the basis of Washington photometry, which has the strongest metallicity sensitivity of any broadband system (Canterna 1976; Geisler et al. 1991). In combination with DDO51, these filters can reliably identify metal-poor giants. We therefore selected a total of 128 candidates, of which 27 were new objects added to the Kepler long cadence (LC) sample in APOKASC GO proposal No. 40033; there was some overlap in the lists of potential metalpoor targets generated with the criteria above. The process for generating the metal-poor star candidate list and the yields from the various methods are described in P. Harding et al. (2014, in preparation). Candidates in any of these three categories are referred to as "halo" in the targeting flags.

\subsubsection{Rapid Rotators}

Rapidly rotating giants (counted as "Rapid Rotators" in Table 1) are relatively rare and may represent interesting stages of stellar evolution, such as recent mergers; we therefore screened the long-cadence sample for signatures of rotational modulation. We found 162 targets with such a signature in the sample.

\subsubsection{Luminous Giants}

Intrinsically luminous giants (defined here as stars with $\log g<2$ ) are under-represented in the public giant sample relative to the field population because they were specifically
Table 1

Breakdown of APOKASC Targets in DR10

\begin{tabular}{lc}
\hline \hline Category & (Non-unique) Number \\
\hline Gold (GOLD) & 286 \\
KASC (KASC) & 678 \\
Halo (HALO) & 40 \\
Luminous giant (LUMINOUS) & 115 \\
Cluster (CLUSTER) & 43 \\
Seismically interesting or outlier & 221 \\
RC (seismically classified) (RC) & 204 \\
RGB (seismically classified) (RGB) & 68 \\
Rapid rotator (ROTATOR) & 17 \\
\hline Total & 1916 \\
\hline
\end{tabular}

selected against in the Kepler planet transit survey design. These stars are important targets for both stellar population and stellar physics studies because they extend the dynamic range in gravity for testing both asteroseismic scaling relationships and the seismic properties of giants. More luminous giants in a magnitude-limited sample will be more distant from both Earth and the Galactic plane than less luminous ones, making the former more likely to be metal-poor. For our spectroscopic program we therefore selected all long-cadence targets with KIC $\log g<1.6$, including $43 \mathrm{M}$ giants and 122 other giants. We also included all long-cadence giants with more than 8 quarters of data and $\log g$ between 1.6 and 2.2, adding 175 additional targets. We also proposed new targets for longcadence observations in a GO program that had four quarters of data (Q14-Q17) that were screened as likely high-luminosity targets which met our magnitude and temperature cuts for APOGEE observation, using KIC properties as a basis. This data set included all stars with KIC $\log g$ between 0.1 and 1.1 and 20 stars randomly selected per 0.1 dex bin in $\log g$ between $\log g$ of 1.1 and 2 . This selection process added 253 new high-luminosity targets in total to the APOGEE sample. All candidates satisfying these criteria were counted as "Luminous Giants" in Table 1.

\subsubsection{Stars with Unusual Pulsation Properties}

Some stars, for poorly understood reasons, have unusually low $l=1$ mode amplitudes (Mosser et al. 2012b; see García et al. 2014a for a discussion), and spectroscopy of such targets is valuable. We included 37 such targets. There are 122 stars with good time coverage and unusually large $\Delta P$, and 15 such targets with unusually small $\Delta P$. Included in our program are 90 stars whose $\Delta P$ values are intermediate between those expected for $\mathrm{RC}$ and RGB stars, which is a possible signature of post-He flash stars (Bildsten et al. 2012). All of these targets were prioritized for spectroscopic observation.

\subsection{Reference Sample Definition and APOKASC Sample Properties}

Our initial target list included all of the stars in the special categories described above. We then defined a reference sample of stars with the highest possible quality of data: this included an accurate classification of evolutionary state and at most one quarter of data missing. We were left with 683 first-ascent giants; they comprise the bulk of the sample. We randomly selected $150 \mathrm{RC}$ and 50 secondary clump stars from a larger pool (1684) of available candidates of comparable quality. We supplemented this list with a secondary priority set (from the Kepler GO program) of targets which otherwise fit our criteria but had less data available. This added 39 first-ascent giants, 

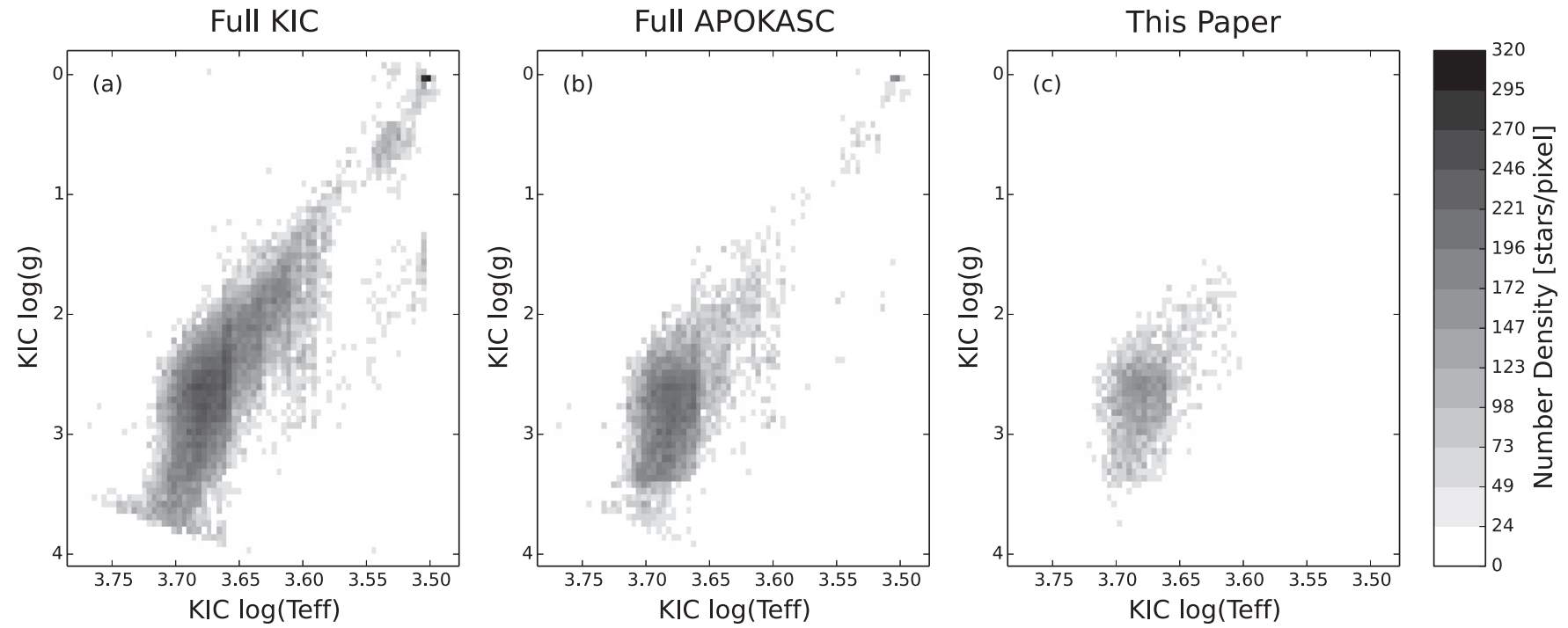

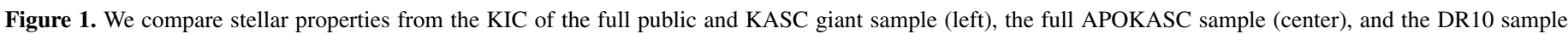
reported in this paper and summarized in Table 1 (right) in the H-R diagram.

58 stars with detected rotation or unusual pulsation properties, 105 secondary RC, and 227 RC stars.

The remainder of the list was filled by public red giants (with or without data on their evolutionary state) and known RC stars, identified as such by the diagnostics discussed in the previous section. The stars with ambiguous evolutionary classification were divided into groups based on their asteroseismically determined $\log g$. Public red giants with ambiguous evolutionary state measurements were considered possible RC stars with $2.35<\log g<2.55$ and possible first-ascent RGB stars otherwise. We allocated 210 slots for RC stars in 21 pointings. If there were fewer than 10 known RC stars in a given target APOGEE pointing, the remainder was filled with possible RC stars. We then selected all of the possible first-ascent RGB stars. These were prioritized first by the number of quarters observed and then randomized among stars with the same length of observations. Then, public giants that appeared as asteroseismic outliers were prioritized by $H$-band magnitude. This was followed by the rest of the known RC stars, prioritized by brightness. Lastly, the public red giants identified as possible RC stars were prioritized first by the number of quarters observed and then randomized among stars with the same length of observations. We illustrate the net effect of our sample selection in Figure 1.

Our final sample is described in Table 1. Some stars are included in more than one category (for example, luminous and halo); and there are 1916 in total. "Gold" refers to the targets used as calibrators for the spectroscopic surface gravity measurements in APOGEE. The full set of targeting flags are included in our main catalog table (see Section 5). In this table we merge the two different labels of SEISMIC INTEREST and SEISMIC OUTLIER into one category, as both classes were targeted because of unusual features in their measured pulsation properties. Stars in the luminous giant category do not have direct targeting flags in subsequent tables, but can be identified by their KIC surface gravity measurements. The targeting flags for each of the other categories are indicated in parentheses in the "Category" column.

There are two particularly important aspects of this sample worth noting. First, a diverse set of objects can be studied with this data set. Second, the overall sample is far from being a simple representation of the underlying stellar population. Basic statistics, such as the ratio of core He-burning to shell H-burning targets, are biased relative to the underlying populations. Not all stars targeted as members of a class are true members. As a concrete example, only a portion of prospective halo giants satisfied our high-resolution abundance criteria. We therefore urge that care be employed when using our data for stellar population studies. The full DR12 sample is both more comprehensive and, arguably, less customized to individual projects; we defer a full discussion of the impact on population studies to the release of the second data set. For completeness, there are three other samples selected in this process and observed in APOGEE that will be released separately because they are either outside of the Kepler fields or do not involve red giants. We observed all Kepler Objects of Interest, or KOIs, that fit our magnitude and color cuts, all dwarfs within our magnitude range that had $T_{\text {eff }}<5500 \mathrm{~K}$, and selected targets observed by the CoRoT satellite (Mosser et al. 2010). A discussion of their properties is beyond the scope of this paper.

\section{SPECTROSCOPIC PROPERTIES}

The APOGEE Stellar Parameters and Chemical Abundances Pipeline (ASPCAP) was employed to infer six atmospheric parameters from the observed spectra: effective temperature $\left(T_{\text {eff }}\right)$, metallicity $([\mathrm{M} / \mathrm{H}])$, surface gravity $(\log g)$, carbon $([\mathrm{C} / \mathrm{M}])$, nitrogen $([\mathrm{N} / \mathrm{M}])$, and $\alpha([\alpha / \mathrm{M}])$ abundance ratios. Automated pipeline analysis is powerful, but there is always the possibility of systematic errors in the derived parameters. Meszaros et al. (2013) therefore checked the spectroscopic measurements of surface gravity, effective temperature, and metallicity against literature values using 559 stars in 20 open and globular clusters and derived corrections to place our results on the same system as these measurements. A limited subset of asteroseismic surface gravities were also used to calibrate results for higher metallicity stars. The net result is two spectroscopic scales: the "raw" pipeline values and the "corrected" ones that were a result of the calibration procedure. Details of the spectroscopic pipeline, its calibration, and the exact equations for the correction terms are presented by Meszaros et al. (2013).

The APOGEE spectroscopic $T_{\text {eff }}$ and metallicity values were used as inputs for deriving the asteroseismic masses, radii, 
surface gravities, and mean densities as described in Section 4. We derived and present results for both the raw and the calibrated spectroscopic scales. In this section of the paper we use the APOKASC sample to provide two new checks on random and systematic uncertainties in the spectroscopic surface gravities and effective temperatures from both asteroseismology and photometry. We compare spectroscopic and asteroseismic surface gravities in our full data set, which is a much larger sample than that used in the Meszaros et al. (2013) work. The Kepler fields are relatively low in extinction, and a complete set of griz and $J H K$ photometry is available for our targets (from the KIC and Two Micron All Sky Survey (2MASS), respectively). Our comparison here of photometric and spectroscopic $T_{\text {eff }}$ measurements, therefore provides a good external check on the KIC extinction map used to derive them and on the absolute spectroscopic temperature scale. Finally, the ASPCAP calibration procedure derived independent corrections to the three major spectroscopic parameters considered here. In principle, one could instead have imposed an external prior on one or more of them, and then searched for a refined spectroscopic solution. Because we have precise asteroseismic surface gravities, we quantify here how our metallicities and temperatures would have been impacted if we had adopted them as a prior rather than independently calibrating all three. A detailed comparison of the full sample results with the KIC and optical spectroscopy, which were not used to calibrate our measurements, is presented in Section 5.

\subsection{The $T_{\text {eff }}$ Calibration}

The ASPCAP spectroscopic effective temperatures were compared with photometric ones using calibrations by González-Hernández \& Bonifacio (2009; hereafter GHB09), using 2MASS $J-K_{s}$ colors (Skrutskie et al. 2006). Meszaros et al. (2013) found systematic differences in the range of 100-200 K between the raw ASPCAP and those derived using the GHB09 scale and literature extinction estimates for star clusters. ASPCAP $T_{\text {eff }}$ values were found to be consistent with literature values from optical spectroscopy, in mild tension with the photometric values. Meszaros et al. (2013) recommended calibrating the ASPCAP $T_{\text {eff }}$ to conform with the photometric scale, because it is closer to the fundamental definition of the effective temperature than the spectroscopic studies; however, the purely spectroscopic scale is defensible, and the difference between the two serves as a measure of plausible system zero-point shifts. The ASPCAP temperatures were corrected between $3500 \mathrm{~K}$ and $5500 \mathrm{~K}$ using an equation derived from the comparison with the GHB09 scale. The GHB09 calibration was chosen as a calibrator because it is explicitly designed for red giants. It is within $30-40 \mathrm{~K}$ of the recent dwarf-only infrared flux method (IRFM) temperature scale of Casagrande et al. (2010). Photometry provides precise relative temperatures for cluster members, and the rms differences between the ASPCAP and photometric temperatures for such stars were used by Meszaros et al. (2013) as a measure of random spectroscopic temperature uncertainties. Random uncertainties ranged from $\sim 200 \mathrm{~K}$ for metal-poor stars to $\sim 100 \mathrm{~K}$ for metal-rich ones.

The majority of the cluster stars used for the calibration are metal-poor, while our sample has a mean metallicity close to solar. It is therefore possible that there could be metallicity trends in the temperature differences, a topic that we explore below when we compare photometric and spectroscopic temperatures in the Kepler fields.

\subsection{The Metallicity Calibration}

The $[\mathrm{M} / \mathrm{H}]$ dimension in ASPCAP was constructed by varying the solar-scaled abundances of all elements except $\mathrm{C}, \mathrm{N}$, and the $\alpha$-capture elements $\mathrm{O}, \mathrm{Mg}, \mathrm{Ca}, \mathrm{Si}, \mathrm{Ti}$. Therefore, the best-fix $[\mathrm{M} / \mathrm{H}]$ represents a line-weighted fit to the iron peak and light odd- $Z$ elements. In practice, as discussed below, $[\mathrm{M} / \mathrm{H}]$ correlates well with $[\mathrm{Fe} / \mathrm{H}]$, and we will treat them as being functionally equivalent in this paper. The ASPCAP metallicity was compared with individual values from high-resolution observations from the literature, and with average cluster values. The derived metallicities from ASPCAP are close to literature values around solar metallicity. The difference in cluster averages between ASPCAP and literature becomes larger than 0.1 dex only below $[\mathrm{M} / \mathrm{H}]=-1$, and this discrepancy increases with decreasing metallicity, reaching $0.2-0.3$ dex around $[\mathrm{M} / \mathrm{H}]=-2$ and lower. An offset of comparable magnitude $(\sim 0.1$ dex $)$ was found above $[\mathrm{M} / \mathrm{H}]=+0.1$. Meszaros et al. (2013) therefore derived a calibration to bring the raw metallicities into agreement with the literature cluster averages.

The metallicity uncertainty was derived from the standard deviation of individual cluster member metallicities around the cluster averages. This scatter can be as high as 0.14 dex for the lowest metallicity globular clusters, but it improves significantly ( similarly to $T_{\text {eff }}$ ) for high metallicities. For open clusters around solar metallicity, the largest scatter is only $0.07 \mathrm{dex}$. We compare our metallicities with those derived from optical spectroscopy and from the KIC in Section 5.

\subsection{The Surface Gravity Calibration}

Surface gravities can be estimated from isochrones for red giants in star clusters if the distances, extinctions, and ages of the systems are known. Meszaros et al. (2013) found significant zero-point offsets between the raw spectroscopic values and those derived from cluster isochrones, motivating an empirical correction. The cluster-based surface gravity calibration was supplemented with a preliminary APOKASC sample of asteroseismic gravities. The Kepler targets are concentrated around solar metallicity, while the cluster sample is predominantly composed of metal-poor systems. We therefore adopted a hybrid empirical calibration that was solely a function of metallicity and solely based on the gold standard asteroseismic surface gravities for stars with $[\mathrm{Fe} / \mathrm{H}]>-0.5$.

This gold standard sample had to be defined prior to the full analysis, and we briefly describe how this sample was assembled and analyzed below. The candidates were selected to be those with the most complete time coverage; see Hekker et al. (2012) for a discussion of the criteria. We then computed the mean asteroseismic parameters using the Hekker et al. (2010) methodology. We adopted effective temperatures based on the griz SDSS filters (Fukugita et al. 1996) from Pinsonneault et al. (2012), using the KIC extinction map. Grid modeling was performed using BaSTI models and adopting the KIC metallicities. We added 0.007 dex in quadrature to the formal uncertainties to account for systematic errors; see Hekker et al. (2013, OCT) for a discussion. A total of 286 stars from this list were observed in DR10 and used as calibrators; see Meszaros et al. (2013) for a more complete discussion. We present the asteroseismic properties used for this calibrating sample in Table 2. We assess the validity of this approach using the full asteroseismic surface gravity from our data below. Table 2 lists the surface gravities derived for the gold standard candidates. These were used to calibrate the ASPCAP spectroscopic surface 

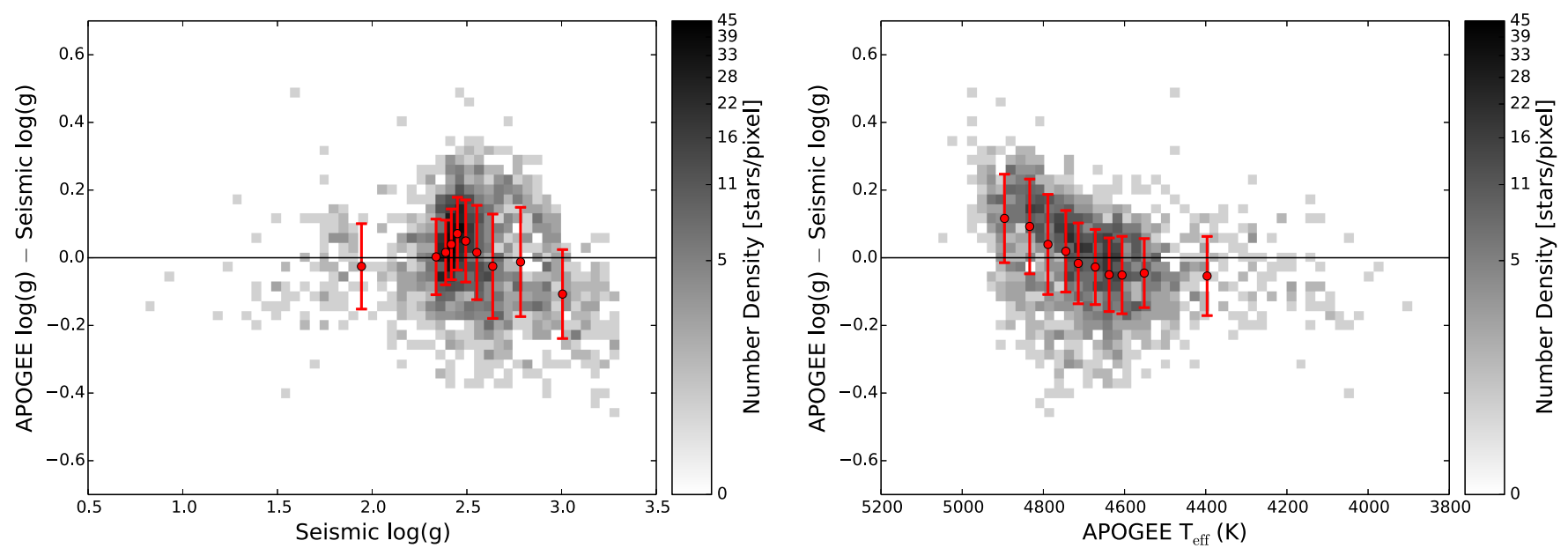

Figure 2. Logarithmic difference between the corrected spectroscopic and asteroseismic surface gravity $\log g$ as a function of asteroseismic log $g$ (left) and spectroscopic $T_{\text {eff }}$ (right) for our full sample. The points with error bars are the means and standard deviations of the data in 10 ranked cohorts of log $g$. The data was divided into 60 bins in $\log g$ (left), $T_{\text {eff }}$ (right), and delta $\log g$ (both), covering the $\log g$ range of $0.5-3.5$, temperature range $3800-5200 \mathrm{~K}$, and gravity difference range -0.7 to +0.7 , respectively. The logarithmic grayscale coding (specified on the right) indicates the number of targets with those properties in the relevant bin.

(A color version of this figure is available in the online journal.)

Table 2

Gold Standard Surface Gravities

\begin{tabular}{lccc}
\hline \hline KIC ID & $\begin{array}{c}T_{\text {eff }} \\
(\mathrm{K})\end{array}$ & {$[\mathrm{Fe} / \mathrm{H}]$} & $\begin{array}{c}\log g \\
(\mathrm{cgs})\end{array}$ \\
\hline 1161618 & 4907 & -0.112 & $2.426 \pm 0.010$ \\
1432587 & 4693 & -0.022 & $1.661 \pm 0.024$ \\
1433593 & 5013 & -0.141 & $2.741 \pm 0.014$ \\
1433730 & 4829 & -0.099 & $2.504 \pm 0.013$ \\
1435573 & 4943 & -0.113 & $2.324 \pm 0.012$ \\
\hline
\end{tabular}

(This table is available in its entirety in a machine-readable form in the online journal. A portion is shown here for guidance regarding its form and content.)

gravities, and are included so that the results of Meszaros et al. (2013) can be replicated. The first column contains the KIC ID. The second is the effective temperature inferred from SDSS photometry in Pinsonneault et al. (2012). The third column is the KIC metallicity, and the fourth is the logarithm (in cgs units) of the surface gravity returned from the OCT pipeline and its uncertainty. We stress that the gravities presented later in the paper supercede these values.

\subsection{Tests of the APOKASC Temperature and Surface Gravity Calibrations}

In the APOKASC sample we have access to extremely precise asteroseismic surface gravities (Hekker et al. 2013), which we adopted for the catalog in preference to the spectroscopic solutions. However, the calibration procedure included only a smaller subset of the data, the gold standard asteroseismic sample, as a reference. Our mean calibrated spectroscopic and asteroseismic $\log g$ values for the full sample are close, with an average offset of 0.005 dex and a dispersion of 0.15 dex. We therefore conclude that our calibration based on the limited preliminary data set yielded reasonable results on average for the full sample (in the sense that the typical differences between our calibrators and spectroscopic values were similar to the same differences for stars not used as calibrators). However, there are interesting underlying trends in the differences between asteroseismic and spectroscopic $\log g$, present in both the gold and full samples, which are illustrated as a function of $\log g$ and $T_{\text {eff }}$ in Figure 2.

We can obtain further insight into the origin of these differences by adding information on evolutionary state. When we do so, a clear division in mean difference emerges between core He-burning, or RC, stars and first-ascent RGB stars (see Figure 3). Some of these differences can be traced to the temperature and surface gravity trends illustrated above, but the differences persist even between members of the same star cluster. We are currently investigating the origin of the gravity offsets between RC and RGB stars. Small differential offsets (at the $5 \%$ level) between asteroseismic radius estimates for $\mathrm{RC}$ and RGB stars were found in NGC 6791 (Miglio et al. 2012) and traced to differences in the sound crossing time at fixed large frequency spacing. However, the impact of the Miglio corrections on the relative radii are too small to explain the observed surface gravity discrepancy, and the differential offset in surface gravity is likely to be at the 0.05 dex level or smaller because of correlations between asteroseismic masses and radii. An offset between the structures of model atmospheres in stars with similar H-R diagram position but with different evolutionary states (and thus differences in mass, helium, or CNO) is in principle possible; however, such effects are expected to be small. We conclude that this offset is an interesting clue that may shed light on both model atmospheres and asteroseismology, but that the mean values of the corrected asteroseismic and spectroscopic gravities are in good agreement.

We can also check on the internal consistency of the spectroscopic temperature scale by comparing our spectroscopic effective temperatures with those that we would have derived using the KIC extinction map and the GHB09 IRFM color-temperature relationship employed in the global spectroscopic calibration. Our results using the KIC extinction map are compared with those in the zero-extinction limit in Figure 4. The dispersion is reasonable at $80 \mathrm{~K}$, but there is a significant zeropoint offset of $-193 \mathrm{~K}$ in the former case. The bulk of the calibrating sample was in metal-poor globular cluster stars, so this feature could reflect a metallicity-dependent offset in the temperature scale; the APOKASC sample is predominantly close to solar abundance. Another possibility is an error in the adopted extinction corrections; as shown above, a zero-extinction 

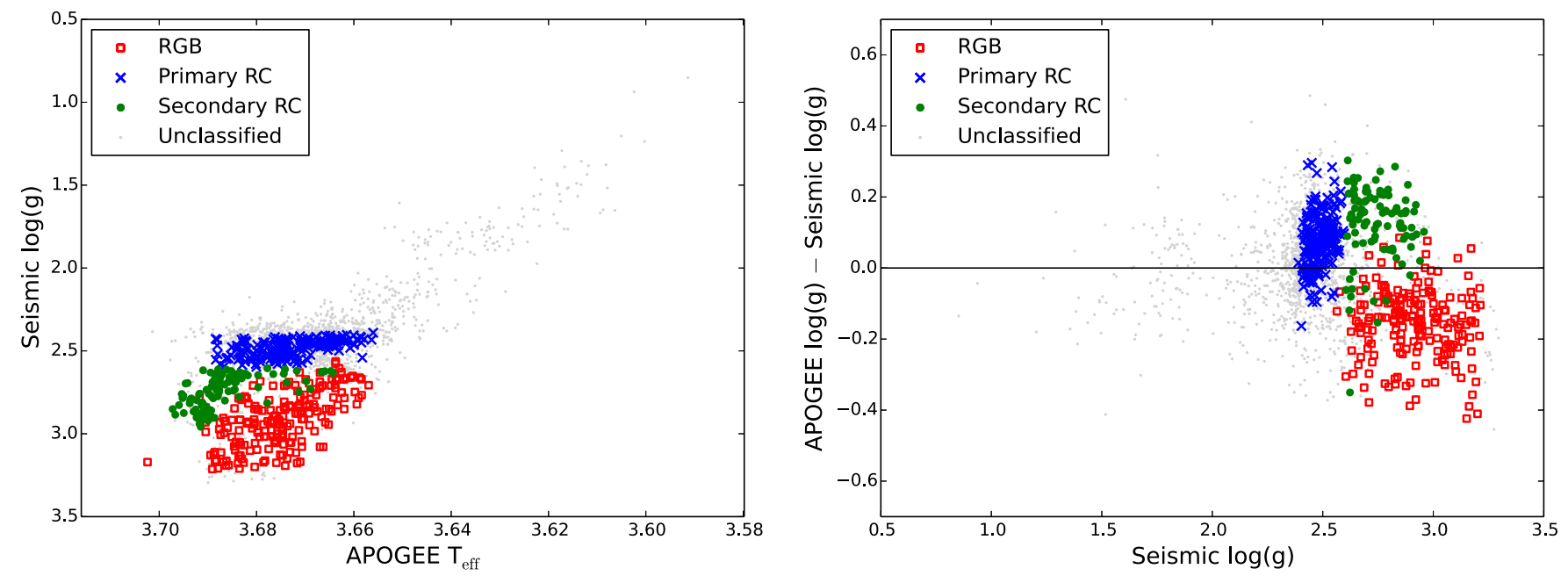

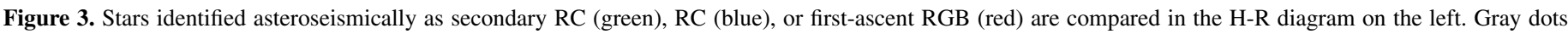

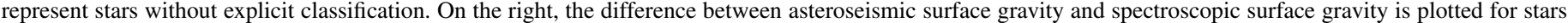
in these evolutionary states as a function of asteroseismic $\log g$. A clear pattern in the differences is visible.

(A color version of this figure is available in the online journal.)
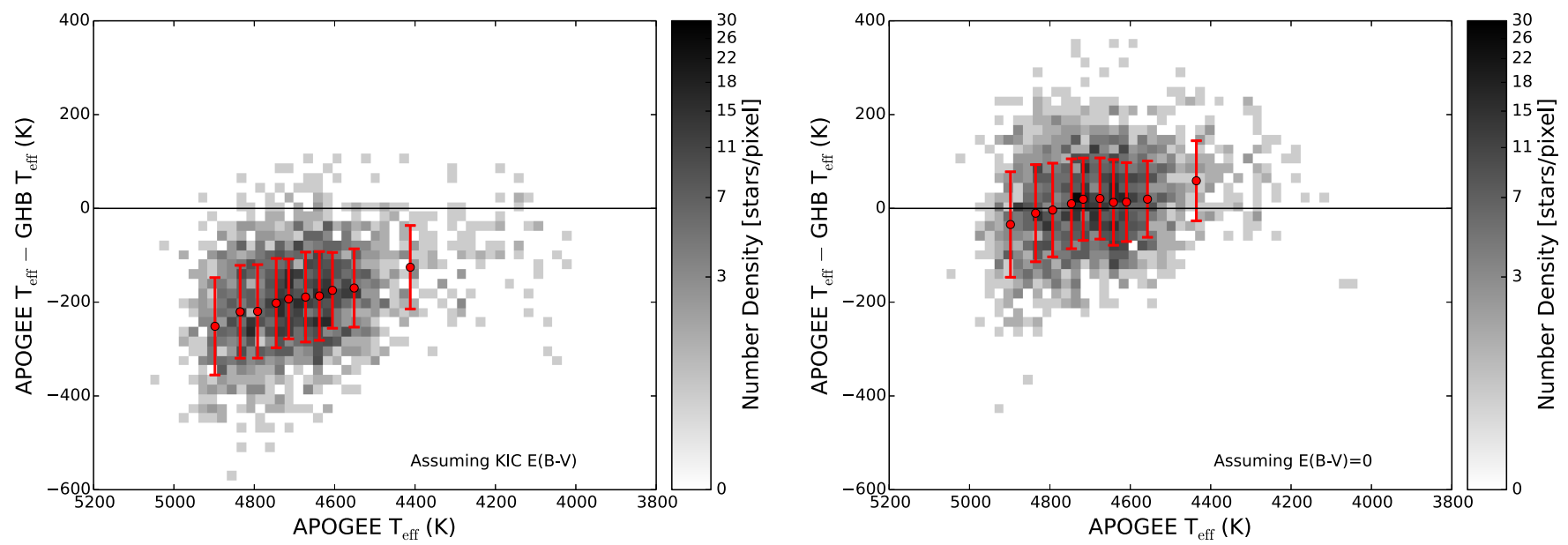

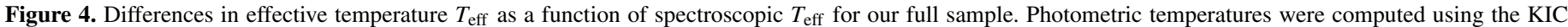

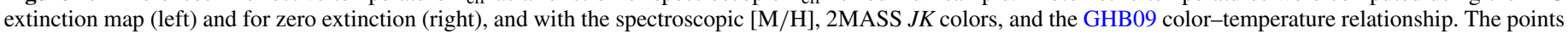

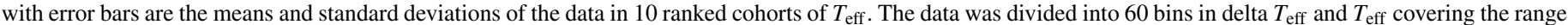

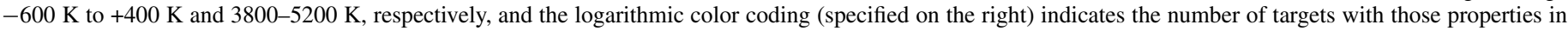
the relevant bin.

(A color version of this figure is available in the online journal.)

case has an average offset of $+11 \mathrm{~K}$. We view this as an unrealistic limit, but the difference between the two certainly highlights the need for an independent re-assessment of the KIC extinction map. Fortunately, a more extensive multi-wavelength data set, especially at longer wavelengths, has been developed since the time the KIC was constructed. Casagrande et al. (2014) used new Stroemgren filter data in a stripe within the Kepler fields, along with an extensive set of literature photometry, to derive systematically smaller extinction values than those in the KIC. Adopting their extinction map would imply a smaller (but real) temperature offset. Zasowski et al. (2013) used 2MASS, IRAC, and WISE data to infer extinctions when developing the APOGEE target list, using the RJCE method (Majewski et al. 2011); work on a related approach for Kepler targets is in progress. Rodrigues et al. (2014) employ a related method of spectral energy distribution fitting and confirm a lower extinction estimate than that obtained from the KIC alone. Adopting the latter extinction values implies a $T_{\text {eff }}$ difference of $74 \mathrm{~K}$ between the corrected ASPCAP and GHB IRFM scale for solar abundance stars.

Finally, we have derived independent calibrations of temperature, metallicity, and surface gravity, applied after the ASPCAP parameter solution was obtained. An alternate method that has been successfully used for dwarfs in the Kepler fields is to search for the best solution adopting the asteroseismic surface gravity as a prior (Chaplin et al. 2014). This approach is similar in philosophy to using isochrone fits to surface gravities in star cluster dwarfs rather than searching for less precise spectroscopic values for warm dwarfs. As a test of adopting this approach, we used the ASPCAP pipeline for all parameters except surface gravity, for which we supplied the asteroseismic values. The chi-squared metric for the best fit was visibly degraded, as expected. However, the resulting metallicities and effective temperatures were also offset from the values obtained from our independent calibration checks. This effect is illustrated in Figure 5 where we compare the values that we would 


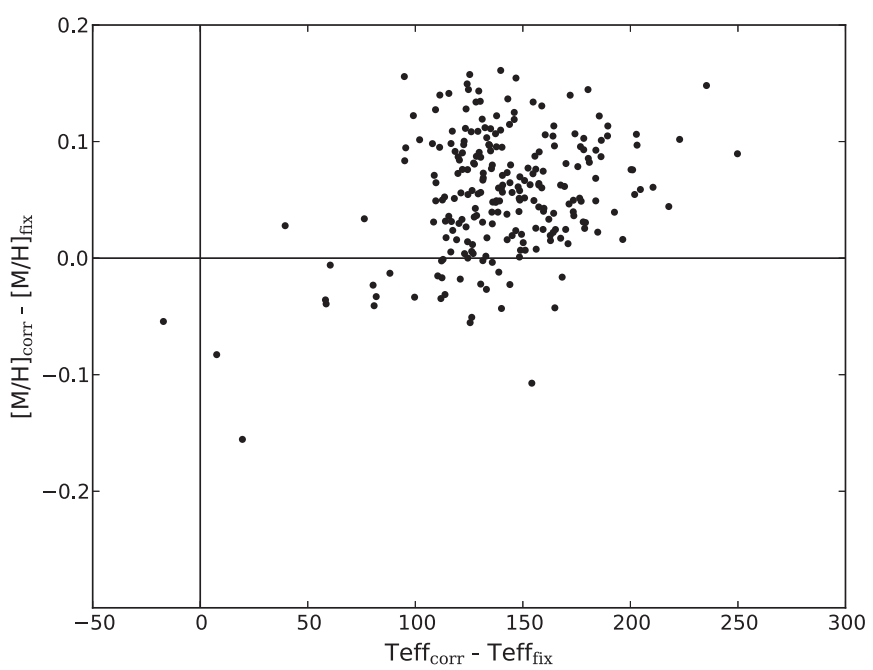

Figure 5. Differences in effective temperature and metallicity between the ASPCAP spectroscopic solution for a fixed asteroseismic surface gravity (subscript fix) and the corrected ASPCAP results (subscript corr). The spectroscopic parameters obtained with an asteroseismic prior are systematically offset to lower metallicity and effective temperature relative to those calibrated against independent diagnostics.

have obtained with an asteroseismic gravity prior with the actual calibrated values. The sense of the difference in temperature is expected from Boltzmann-Saha balance considerations, and the corrected values using this approach are actually in worse agreement than the raw ones when compared with independent measurements. We therefore conclude that the Meszaros et al. (2013) approach of independent calibrations for each of the spectroscopic parameters is more accurate for our purposes than adopting an asteroseismic surface gravity prior.

In summary, our raw spectroscopic parameters have been derived using a homogeneous analysis method. Comparisons with independent measurements motivated us to define modest correction terms for metallicity and effective temperature and more substantial ones for the spectroscopic surface gravities. At the metal-rich end, the spectroscopic surface gravities were tied to an asteroseismic reference scale using a limited sample of gold standard targets. With the full DR10 sample of spectroscopic and asteroseismic data we revisited the spectroscopic surface gravity calibration with a much larger sample of asteroseismic surface gravities. The larger asteroseismic sample is in good mean agreement with the Meszaros et al. (2013) calibration, but there are modest (but real) systematic offsets at the $\sim 0.1$ dex level between the asteroseismic and spectroscopic scales as functions of effective temperature, gravity, and evolutionary state. Further work is needed to identify the origin of these effects (in terms of systematics in either the asteroseismic or spectroscopic surface gravities).

We also compared our spectroscopic effective temperatures for Kepler field red giants against $T_{\text {eff }}$ derived from the same photometric temperature calibration that was used in star clusters. There is also an offset of $193 \mathrm{~K}$ between the spectroscopic $T_{\text {eff }}$ scale and the IRFM photometric $T_{\text {eff }}$ scale if the KIC extinction map is adopted; however, there is independent evidence that the KIC extinctions are overestimated, so this result should be treated as an upper bound on systematic $T_{\text {eff }}$ errors for our sample. With the extinction map of Rodrigues et al. (2014) we can quantify the zero-point shift more precisely (and it is at the $74 \mathrm{~K}$ level). If this offset is confirmed, it implies a metallicitydependent temperature correction that was not captured in the original calibration. We view these temperature and gravity comparisons as fair indicators of potential systematic uncertainties in these properties. Neither of these comparisons directly address the accuracy and precision of our metallicity estimates, which we discuss in Section 5 when comparing our final catalog values with the KIC and optical spectroscopy.

\section{ASTEROSEISMIC PROPERTIES}

Our goal for the asteroseismic sample was to provide masses, radii, and surface gravities for all of our red giants. This is a complex task with multiple stages, and we discuss our methodology below; we begin with a brief summary.

We processed and corrected the raw light curves to extract the oscillation frequencies. We then identified the modes, and distilled the information from the observed oscillation pattern down to two global asteroseismic parameters: the frequency of maximum power and the average large frequency spacing, whose interpretation is discussed below. These parameters, combined with the effective temperature, could in principle be used to solve for the asteroseismic masses and radii with the aid of scaling relations. We took the additional step of combining information from stellar models with the global asteroseismic parameters, and used this grid modeling effort to both identify outliers and to refine our estimates. In all cases we employed multiple methods and made an attempt to assess random and systematic uncertainties. We release asteroseismically determined stellar parameter estimates for two distinct spectroscopic inputs, corresponding to the raw and corrected scales discussed above.

\subsection{Light Curve Data Analysis}

Preparation of Kepler long-cadence data (Jenkins et al. 2010) for asteroseismic analysis was handled in three cohorts. We used Pre-search Data Conditioning Simple Aperture Photometry (PDC-SAP) light curves (Smith et al. 2012; Stumpe et al. 2012) for a cohort of 2067 field stars. These data were prepared for asteroseismic analysis in the manner described by García et al. (2011). Kepler data for quarters Q0 through Q8 were used for these targets. A second cohort of 657 field stars had pixel-level data available from Kepler observing quarters Q0 through Q12 inclusive. Aperture photometry was performed on these data (S. Mathur et al., in preparation), producing light curves that were more stable at longer timescales than the PDC-SAP data. This improvement was at the expense of having slightly elevated high-frequency noise. Finally, raw Q0 through Q11 data were prepared for 34 stars in the open cluster NGC 6819, again using the procedures described in García et al. (2011; see also Stello et al. 2011a). A total of 581 stars had data available in both the pixel and the public sets described above (a point we return to briefly at the end of Section 4.2.3).

In total, five data analysis methods-Huber et al. (2009), Hekker et al. (2010), Kallinger et al. (2010), Mathur et al. (2010), and Mosser et al. (2011b) -were used to extract independent estimates of two global asteroseismic parameters from the frequency-power spectrum of the light curves. Some of the analysis methods were not applied to every data set. One parameter was the average large frequency separation, $\Delta v$, the mean spacing between consecutive overtones of the same angular degree, $l$. The average large separation scales to very good approximation as $\rho^{1 / 2}, \rho \propto M / R^{3}$ being the mean density of a star having mass $M$ and surface radius $R$ (e.g., see Tassoul 1980; Ulrich 1986; Christensen-Dalsgaard 1993). 
The dependence of $\Delta v$ on the mean stellar density may be used as a scaling relation normalized by solar properties and parameters, i.e.,

$$
\frac{\Delta v}{\Delta v_{\odot}} \simeq \sqrt{\frac{M / M_{\odot}}{\left(R / R_{\odot}\right)^{3}}} .
$$

The second global parameter is $v_{\max }$, the frequency of maximum oscillation power. It has been shown to scale to good approximation as $g T_{\text {eff }}^{-1 / 2}$ (Brown et al. 1991; Kjeldsen \& Bedding 1995; Chaplin et al. 2008; Stello et al. 2009a; Belkacem et al. 2011), where $g$ is the surface gravity and $T_{\text {eff }}$ is the effective temperature of the star. The following scaling relation may therefore be adopted:

$$
\frac{v_{\max }}{v_{\max , \odot}} \simeq \frac{M / M_{\odot}}{\left(R / R_{\odot}\right)^{2} \sqrt{\left(T_{\mathrm{eff}} / T_{\mathrm{eff}, \odot}\right)}} .
$$

The completeness of the results (i.e., the fraction of stars with returned estimates) varied, since some pipelines are better suited to analyzing different ranges in $v_{\max }$.

We selected one data analysis method, OCT (Hekker et al. 2010), to provide the catalog global asteroseismic parameters of the stars in all three cohorts. This selection was based on the returned $v_{\max }$ values. The Hekker et al. method had the highest completeness fraction, and results that were consistent with those given by the other pipelines. This approach ensured we obtained a homogeneous set of global asteroseismic parameters, which were then used to estimate the fundamental stellar properties (see Section 4.2 below).

For outlier rejection we selected a reference method for each cohort, this being the one whose $v_{\max }$ estimates lay closest to the median over all stars in that cohort (Mosser et al. for the pixel and public cohorts; and Kallinger et al. for the cluster cohort). If the Hekker et al. $v_{\max }$ differed from the reference $v_{\max }$ by more than $10 \%$, we rejected the asteroseismic parameters for that star. This procedure removed 28 stars from the pixel cohort, 167 stars from the public cohort, and 1 star from the cluster cohort.

Uncertainties on the final $\Delta v$ and $v_{\max }$ of each star were obtained by adding, in quadrature, the formal uncertainty returned by the Hekker et al. method to the standard deviation of the values returned by all methods. We also allowed for known systematic errors in Equation (1) (e.g., see White et al. 2011; Miglio et al. 2012), by including an additional systematic contribution of $1.5 \%$ (also added in quadrature). Because $\Delta v$ is usually determined more precisely than $v_{\max }$, we also added the same systematic contribution to the $v_{\max }$ uncertainties. This is essentially the approach adopted by Huber et al. (2013) in their analysis of asteroseismic KOIs.

\subsection{Grid-based Modeling}

For each star we used the two global asteroseismic parameters, $\Delta v$ and $v_{\max }$, together with the estimates of effective temperature $T_{\text {eff }}$ and metallicity $[\mathrm{Fe} / \mathrm{H}]$, as input to "grid-based" estimation of the fundamental stellar properties. This approach matches the set of observables to theoretical sets calculated for each model in an evolutionary grid of tracks or isochrones. The fundamental properties of the models (i.e., $R, M$, and $T_{\text {eff }}$ ) were used as inputs to the scaling relations (Equations (1) and (2)) to calculate theoretical values of $\Delta v$ and $v_{\max }$ for matching with the observations.

Every pipeline adopted solar values $\Delta v_{\odot}=135.03 \mu \mathrm{Hz}$ and $v_{\max , \odot}=3140 \mu \mathrm{Hz}$, which are the solar values returned by the pipeline we selected to return final values on our sample (Hekker et al. 2010). The uncertainties in $\Delta v_{\odot}(0.1 \mu \mathrm{Hz})$ and $v_{\max , \odot}$
$(30 \mu \mathrm{Hz})$ were accounted for by increasing the uncertainties in the $\Delta v$ and $v_{\max }$ data of each star, using simple error propagation. Further details on grid modeling using asteroseismic data may be found in, for example, Stello et al. (2009b), Basu et al. (2010, 2012), Gai et al. (2011), and Chaplin et al. (2014).

We adopted a grid-based analysis that coupled 6 pipeline codes to 11 model grids, comprising a selection of widely used sets of stellar evolution tracks and isochrones that have a range of commonly adopted input physics. In applying several gridpipeline combinations, we capture implicitly in our final results the impact of model dependencies from adopting different commonly used grids, and differences in the detail of the pipeline codes themselves.

\subsubsection{Grid Pipelines}

Grid-based estimates of the stellar properties were returned by the following pipeline codes.

1. The Yale-Birmingham (YB; Basu et al. 2010, 2012; Gai et al. 2011);

2. The Bellaterra Stellar Properties Pipeline (BeSPP; Serenelli et al. 2013 extended for asteroseismic analysis);

3. PARAM (da Silva et al. 2006; Miglio et al. 2013);

4. RADIUS (Stello et al. 2009b);

5. AMS (Hekker et al. 2013); and

6. The Stellar Fundamental Parameters (SFP) pipeline (Kallinger et al. 2010; Basu et al. 2011).

The YB pipeline was used with five different grids: models from the Dartmouth group (Dotter et al. 2008) and the Padova group (Marigo et al. 2008; Girardi et al. 2000), the set of YY isochrones (Demarque et al. 2004), a grid constructed using the Yale Stellar Evolution Code (YREC; Demarque et al. 2008) and described by Gai et al. (2011; we refer to this set as YREC), and another set of models constructed with a newer version of YREC with updated input physics (we refer to this grid as YREC2) that has been described by Basu et al. (2012). The Dotter et al. and Marigo et al. grids include models of RC stars; YREC and YREC2 include only models of He-core burning stars of higher mass, which do not go through the He flash; while YY has no RC models. The BeSPP pipeline was run with two grids. The first grid is comprised of models constructed with the GARSTEC code (Weiss \& Schallt 2008) and the parameters of the grid are described in Silva Aguirre et al. (2012). The second grid is comprised of the BaSTI models of Pietrinferni et al. (2004), computed for use in asteroseismic studies (see Silva Aguirre et al. 2013). Both grids include RC models. RADIUS was coupled to a grid constructed with the ASTEC code (Christensen-Dalsgaard et al. 2008), as described in Stello et al. (2009b) and Creevey et al. (2012), which does not include RC models.

The codes above were all employed in the grid-based analysis of solar-type Kepler targets described in Chaplin et al. (2014), where summary details of the physics employed in the grids may also be found.

PARAM was run using a grid comprising models of the Padova group (Marigo et al. 2008), again including RC stars; further details may be found in Miglio et al. (2013). AMS is based on an independent implementation of the YB pipeline, and was run using the BaSTI models of Pietrinferni et al. (2004). The SFP pipeline was also coupled to BaSTI models. These grids include RC models.

For this first analysis of the APOKASC red giants an asteroseismic classification (i.e., RGB or RC) was not available 

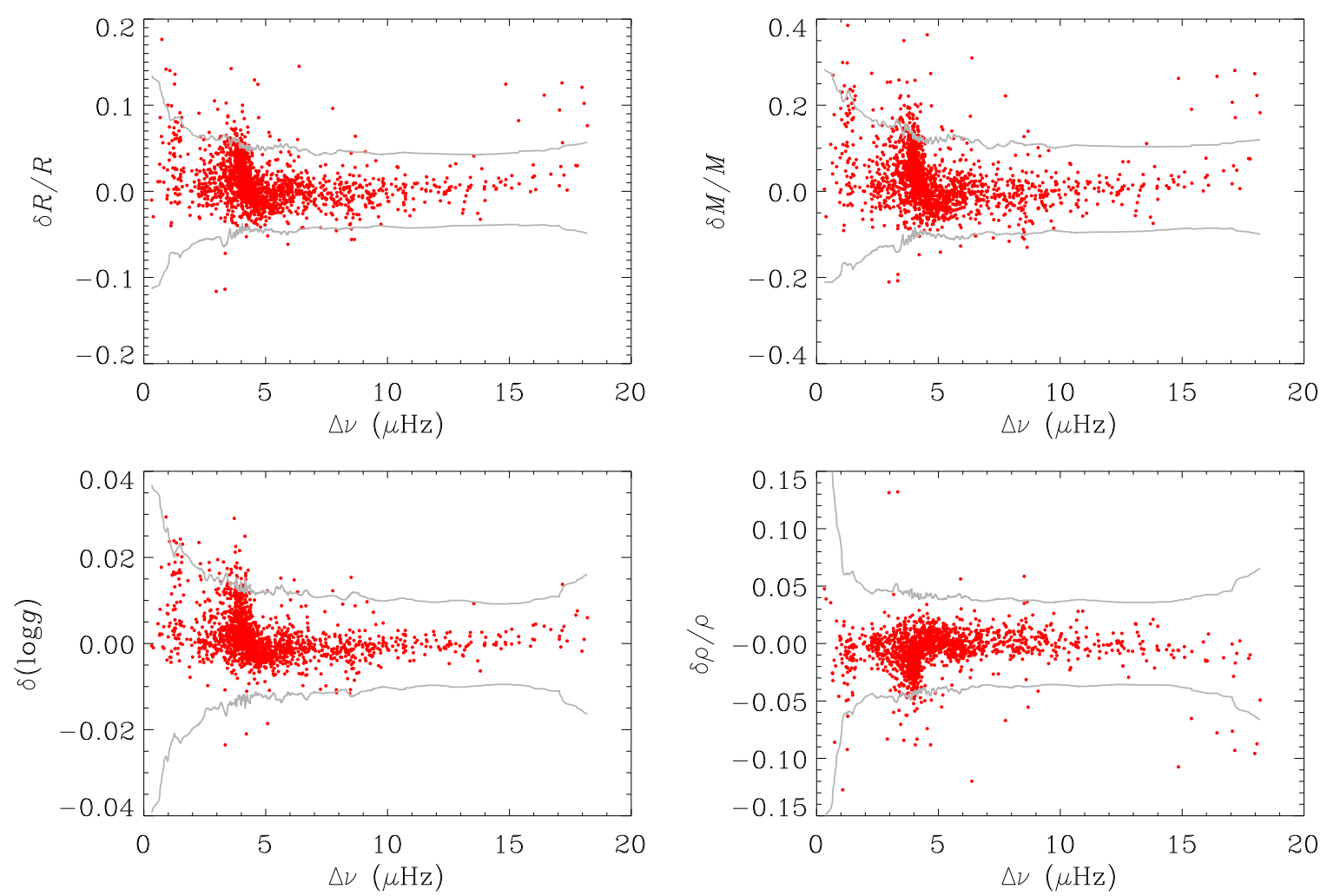

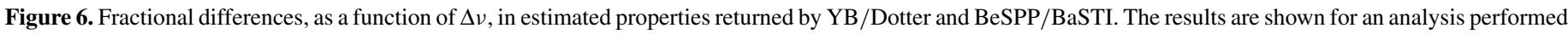

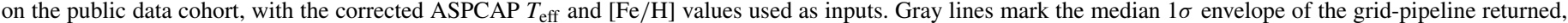
formal uncertainties. These lines are included to help judge the typical precision only.

(A color version of this figure is available in the online journal.)

for many of the stars. Therefore, no a priori categorization information was used in the grid-based searches. Although the cohort evidently contains many RC stars, we nevertheless obtained some results using grids comprised of only RGB models, to test the impact of neglecting the RC. However, as explained below, our final results are produced using only those grids that included RC models.

\subsubsection{Results from Grid-based Analyses}

Figure 6 is an example of the typical differences we see in the estimated properties returned by different grid-pipeline combinations, here those between YB/Dotter and BeSPP/ $\mathrm{BaSTI}$ (the latter chosen as the reference). Both sets comprise results from grids that included RC models. Results are plotted from stars in the public data cohort, with $\Delta v, v_{\max }$, the revised ASPCAP $T_{\text {eff }}$, and $[\mathrm{Fe} / \mathrm{H}]$ values used as inputs. We plot fractional differences in $R, M$, and $\rho$ and absolute differences in $\log g$. Gray lines mark envelopes corresponding to the median of the $1 \sigma$ uncertainties returned by all grid pipelines. Medians were calculated in 10 target batches sorted on $\Delta v$. These lines are included to help judge the typical precision only; uncertainties in the results of individual targets may of course be slightly different. Similar trends to those present here are seen in results on the pixel and cluster target cohorts, and in results from using the raw ASPCAP $T_{\text {eff }}$ and $[\mathrm{Fe} / \mathrm{H}]$ scales as inputs.

On the whole, the differences lie within the median formal uncertainty envelopes, which is encouraging, i.e., the scatter between different grid-pipeline combinations tends to be smaller than the typical intrinsic, formal uncertainties returned by those pipelines. However, we do see clear excess scatter centered on $\Delta v \simeq 4 \mu \mathrm{Hz}$, which corresponds to the location of $\mathrm{RC}$ stars. A significant fraction of our target sample lies in this region. Further analysis presented below suggests that this is genuine extra scatter, and not a sampling effect (see Figure 8 in Section 4.2.3 and accompanying discussion). The presence of this scatter evidently reflects the difficulty of discriminating between RC and RGB models when no a priori categorization is used as input, as was the case here.

Differences with respect to the reference results of BeSPP/ BaSTI tend, not surprisingly, to be more pronounced for grids which did not include RC models. Grid-pipeline combinations with no RC models appear to compensate for the absence of the clump by the inclusion of high-mass (lower-age) RGB solutions, which are not present in the RC sets. Although at the lowest masses the results for grids with and without RC models are similar, the mapping to age is of course different: grid-pipeline combinations with no RC models yield older solutions at the same mass.

The lack of a priori information on the evolutionary state has significant implications for our ability to return not only robust estimates of the absolute ages, but even accurate measures of the relative ages of the cohort, i.e., the relative chronology will be scrambled if an RC star is incorrectly matched to a model of an RGB star, or vice versa. Indeed, the problem is even more subtle. The grid-based codes compute a likelihood for every model that is a reasonable match (within several sigma) to the observables. Estimated properties are returned from the distributions formed by these likelihoods, i.e., the analysis is probabilistic in nature. Without information on the evolutionary state, the distribution functions may be comprised of a mix of RC and RGB information. That is why, for now, we do not provide ages explicitly in the catalog (although the reader may compute 

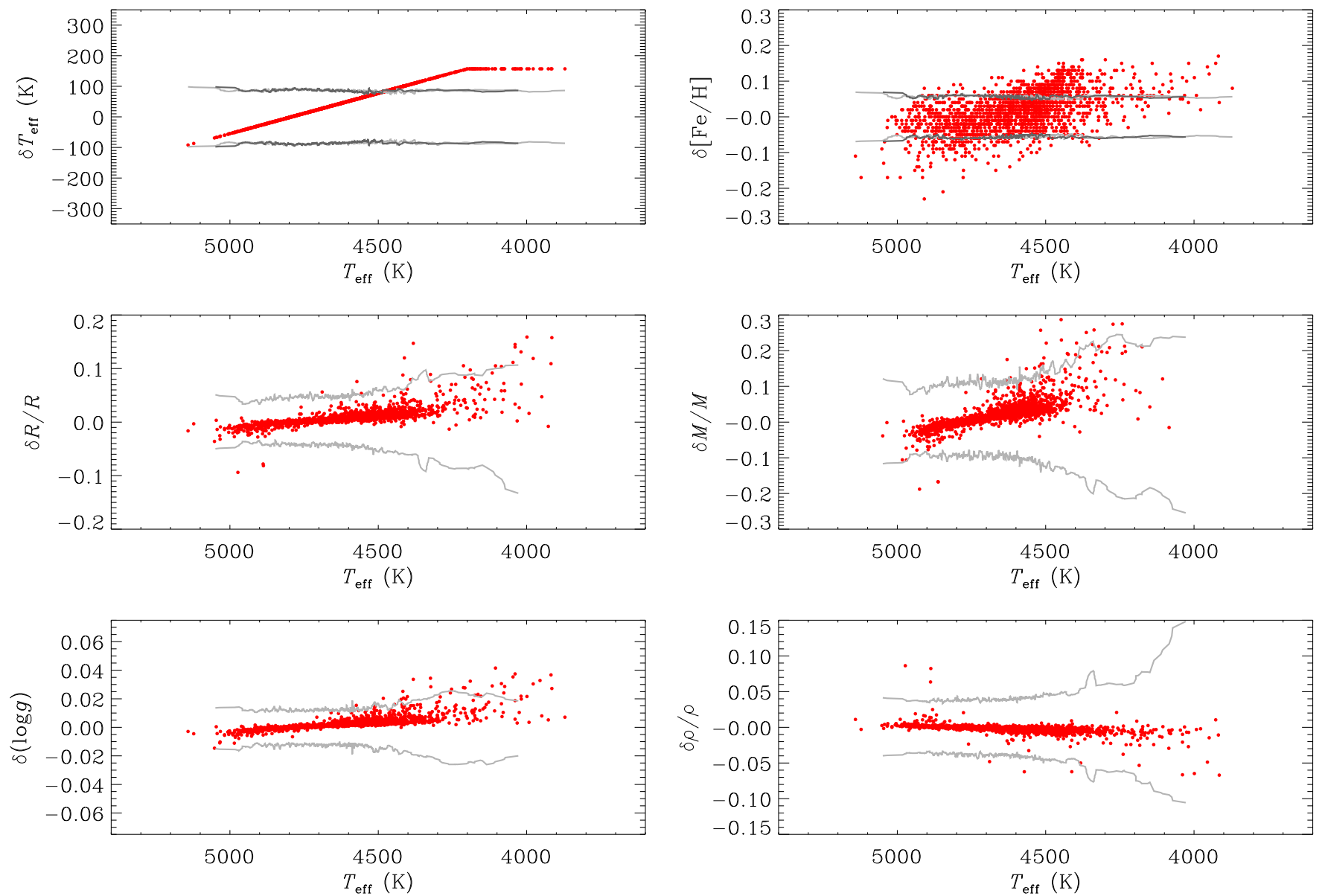

Figure 7. Fractional differences in estimated properties returned by BeSPP/BaSTI, for analyses performed on the public data cohort with the two different $T_{\text {eff }}$ and $[\mathrm{Fe} / \mathrm{H}]$ inputs. Differences are defined as results with corrected ASPCAP scale inputs minus results with raw ASPCAP scale inputs. Gray lines mark the median $1 \sigma$ envelope (over all pipelines) of the returned formal uncertainties. The top panels show the absolute $T_{\text {eff }}$ and $[\mathrm{Fe} / \mathrm{H}]$ differences between the two sets of input parameters, while the bottom four panels record the impact on the derived asteroseismic properties. The impact on the separate mass and radius measurements (in the middle panels) is larger than the impact on the surface gravity and mean density (in the bottom panels).

(A color version of this figure is available in the online journal.)

their own ages from the masses and metallicities provided). Bedding et al. (2011) noted that the oscillation spectra of the giants can be used to distinguish between RC and RGB stars, and we discussed methods earlier in the text that could provide automated estimation of evolutionary state for many targets in the sample. Unfortunately, our initial screening procedure only produced evolutionary state diagnostics for $\sim 25 \%$ of the targets. We are in the process of developing more efficient tools that will provide measurements for an even larger fraction of the sample. Thus although we do not have the information now, the desired evolutionary information will be available to help construct the next version of the catalog.

Figure 7 shows the impact on the public data cohort results of switching from one set of ASPCAP $T_{\text {eff }}$ and $[\mathrm{Fe} / \mathrm{H}]$ inputs to the other. Results for the pixel and cluster cohorts show similar trends. The top left-hand panel plots the corrected ASPCAP $T_{\text {eff }}$ minus the raw ASPCAP $T_{\text {eff }}$, showing the piecewise correction that was applied to the raw temperatures to yield the corrected scale. The lines follow the median $1 \sigma$ envelopes of the uncertainties. The top right-hand panel presents the corrected ASPCAP $[\mathrm{Fe} / \mathrm{H}]$ minus the raw ASPCAP $[\mathrm{Fe} / \mathrm{H}]$. The other panels display the fractional differences in estimated properties returned by BeSPP/BaSTI, in the sense corrected ASPCAP minus raw ASPCAP. As in the previous figures, gray lines mark the median $1 \sigma$ envelopes (over all pipelines) of the returned, formal uncertainties.

With reference to the asteroseismic scaling relations, the trends revealed in Figure 7 may be understood largely in terms of the changes to the temperature scale. The relations imply that, all other things being equal, $M \propto T_{\text {eff }}^{1.5}, R \propto T_{\text {eff }}^{0.5}$, and $g \propto T_{\text {eff }}^{0.5}$, while the seismic estimates of $\rho$ are not affected by the change to $T_{\text {eff }}$. The plotted property differences are thus seen to reflect, to good approximation, the trend in $T_{\text {eff }}$, although this clearly does not explain all the differences, i.e., there is also the impact of the changes in $[\mathrm{Fe} / \mathrm{H}]$ to consider (which are, for example, apparent in the small differences seen in the estimates of $\rho$ ).

\subsubsection{Asteroseismic Catalog Properties and Uncertainties}

We provide tables of estimated properties for each of the raw ASPCAP and corrected ASPCAP scales (see Tables 4 and 5 below). For both sets of inputs, the properties in the catalog are those that were returned by $\mathrm{BeSPP} / \mathrm{BaSTI}$. Its results lay closest to the median over all grid-pipelines and targets. By choosing one grid-pipeline to provide the final properties we avoid mixing results that are subject to different input physics and pipeline methodology. We instead opted to reflect those differences in the quoted final uncertainties, by taking into 

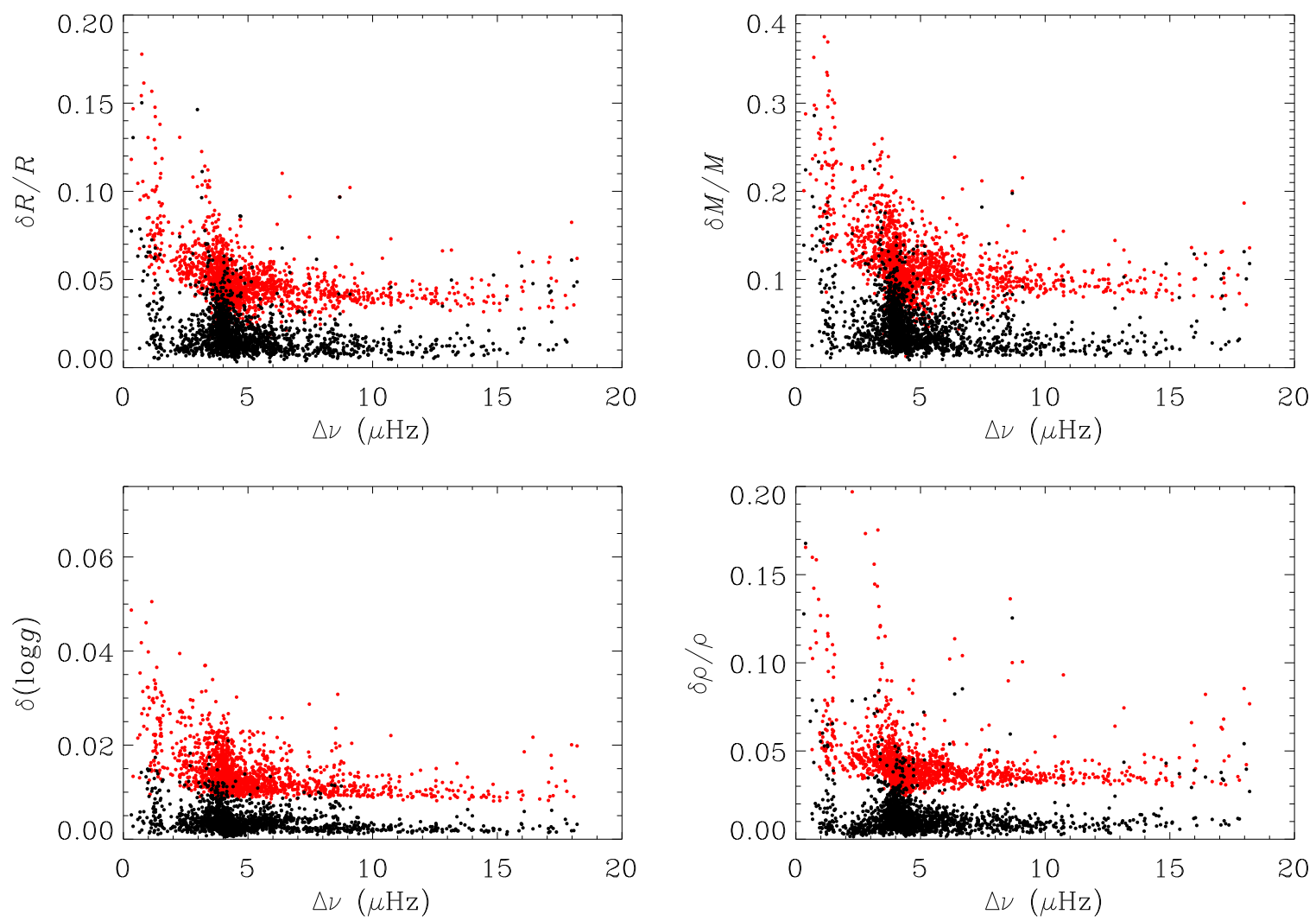

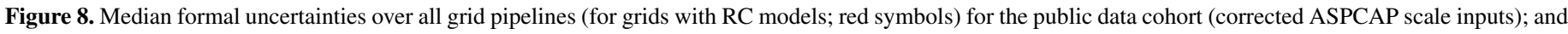

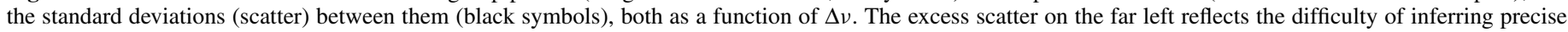
asteroseismic properties for luminous giants, while the excess scatter close to $4 \mu \mathrm{Hz}$ is caused by ambiguity between RC and RGB solutions.

(A color version of this figure is available in the online journal.)

account the scatter between results returned by the different gridpipeline combinations. Our approach is therefore similar to that adopted by Chaplin et al. (2014) for asteroseismic dwarfs and subgiants. We emphasize that in this consolidation we used only results from grids that included RC models. The BeSPP/BaSTI models did not include convective overshoot or semi-convection and incorporated mass loss with an efficiency $\eta=0.4$. These choices did not have a major impact on the derived masses and radii.

Figure 8 plots the median formal uncertainties returned by grid-pipeline combinations including $\mathrm{RC}$ models only (red symbols), again for results from the public data cohort using the corrected ASPCAP scale inputs. For each property of every star we calculated the standard deviation of the results returned by the various grid-pipeline combinations. These estimates of the scatter are plotted in black. The median standard deviations are approximately $4 \%$ in mass, $1 \%$ in radius, 0.003 dex in $\log g$, and $1 \%$ in density.

The plots demonstrate clearly that the scatter between the grid-pipelines is typically smaller than the formal uncertainties returned by any one pipeline. Moreover, both the scatter and the formal uncertainties are larger at the $\Delta v$ where we find RC stars. This result again emphasizes the extra challenges posed for the grid searches in this part of the parameter space in the absence of an a priori categorization.

The above measures of scatter were combined in quadrature with the individual formal uncertainties returned by BeSPP/ BaSTI to yield the final uncertainties on the estimated properties. Distributions of the scatter between pipelines are shown in Figure 9. The plotted histograms (here, for the same cohort of results as the previous figures) were constructed by computing residuals for each pipeline grid with respect to BeSPP/BaSTI, and normalizing each residual by the median property uncertainty given by the pipelines for that star. We then accumulated residuals for all stars in the cohort, and binned the residuals to give the plotted "super distributions." The most striking aspect of all the histograms is their Gaussian-like appearance (see similar results for solar-type stars in Chaplin et al. 2014). Our median final uncertainties are approximately $12 \%$ in mass, $5 \%$ in radius, $0.01 \mathrm{dex}$ in $\log g$, and $3 \%$ in density. These uncertainties will typically be higher for those stars which have $\Delta v \simeq 4 \mu \mathrm{Hz}$ (i.e., RC stars).

Finally, some targets appeared in both the pixel and publicdata samples. In such cases we adopted the properties estimated from the higher-quality, longer pixel light curves to be the catalog properties. Estimated properties for the cluster stars all came from analyses of the cluster data.

A question that might be legitimately be asked is why should we resort to grid-based modeling, which makes our results model dependent, when the direct use of Equations (1) and (2) could, in principle, produce model-independent results. The issue is that the scaling relations assume that all values of $T_{\text {eff }}$ are possible for a star of a given mass and radius and are unconstrained by the equations of stellar structure and evolution. Thus it is quite possible that observational errors in $T_{\text {eff }}, \Delta v$, and $v_{\max }$ can produce estimated values of mass and radius inconsistent with the theory of stellar structure and evolution. The grid-based method takes this constraint into account implicitly, as well as including metallicity information not used in the scaling relations. 

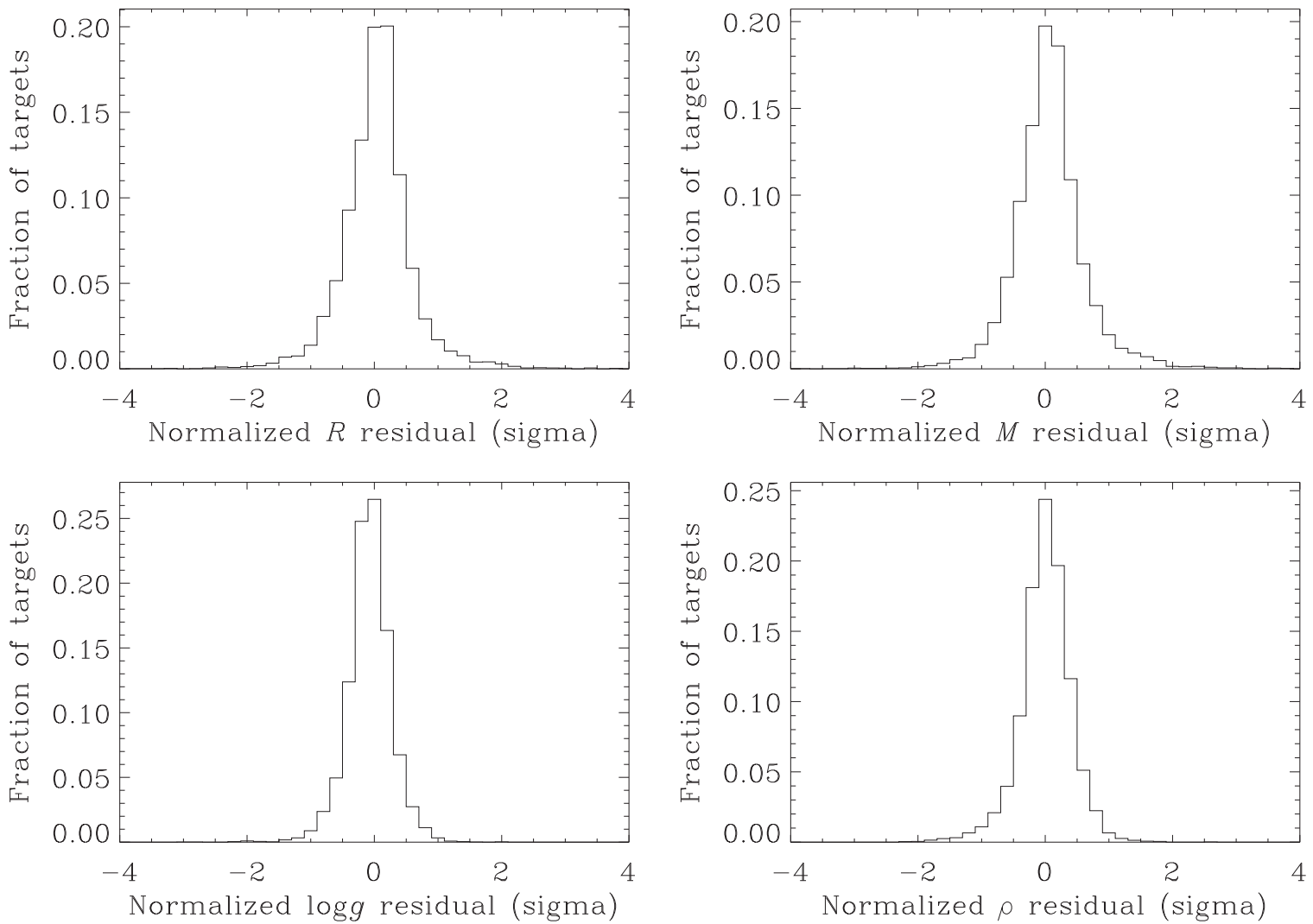

Figure 9. Histograms, for each property, of uncertainty-normalized residuals over all pipeline-grid combinations (omitting BeSPP/BaSTI) and all stars in the pubic data cohort (corrected ASPCAP scale inputs). The plotted residuals were calculated with respect to the BeSPP/BaSTI results.
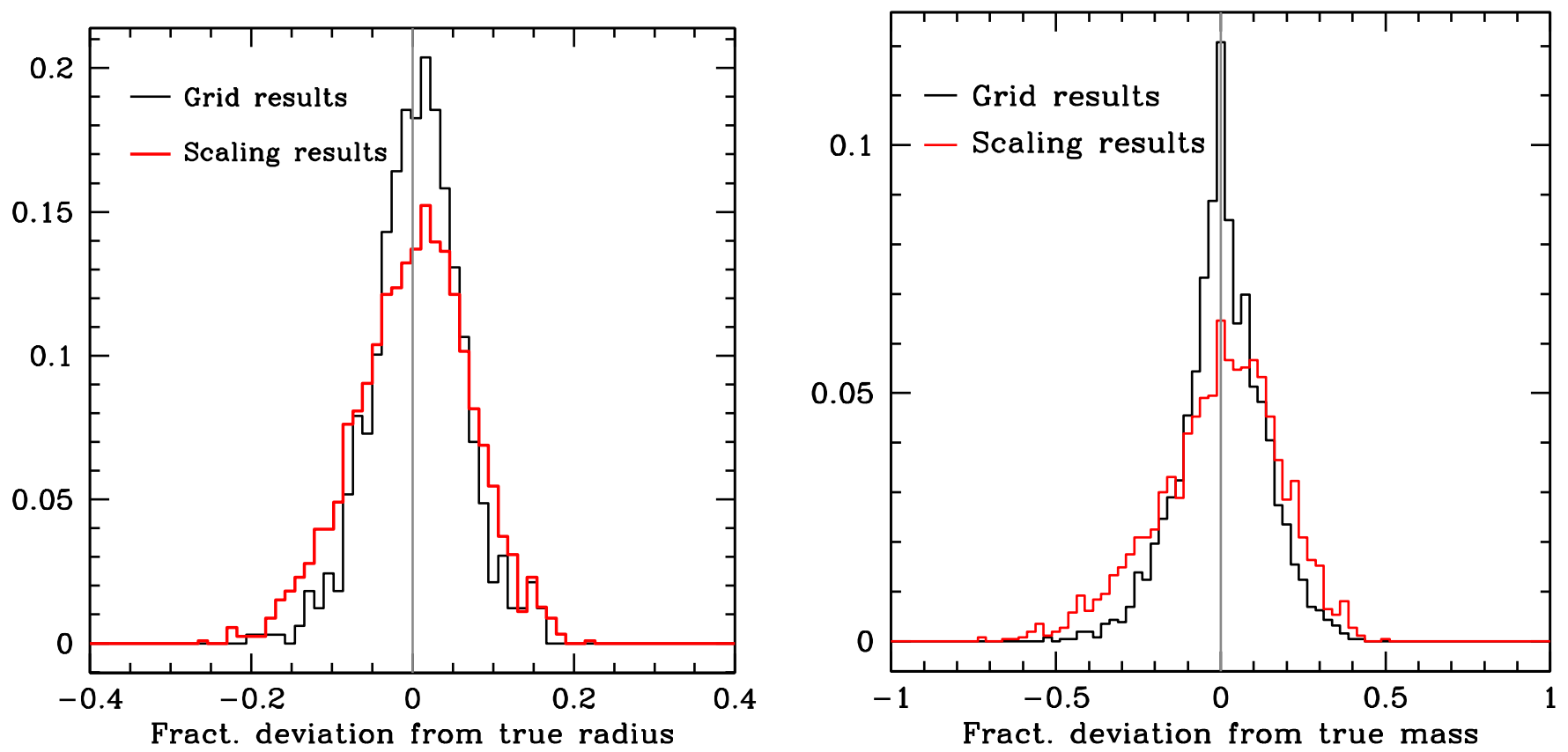

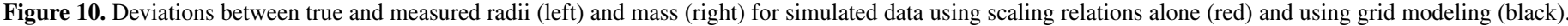
The simulated data set is taken from Gai et al. (2011).

(A color version of this figure is available in the online journal.)

Properties of asteroseismic grid-modeling results have been studied in detail by Gai et al. (2011). They showed that propagated errors are in general smaller in grid-based results than those inferred from propagating scaling relations. The Gai et al. work dealt with simulated stars over a large part of the $\mathrm{H}-\mathrm{R}$ diagram, and the gain obtained by grid-based modeling may not persist in the narrow temperature range occupied by red giants. To test this hypothesis, we have taken the Gai et al. simulated sample and selected all objects with $\Delta v<20 \mu \mathrm{Hz}$ as likely red giants. In Figure 10 we show histograms of the fractional difference between the true and inferred radius and mass for these stars obtained with both the scaling relations 
Table 3

APOKASC Catalog Basic Data

\begin{tabular}{|c|c|c|c|c|c|c|c|c|c|c|}
\hline KIC ID & 2MASS ID & R.A. & Decl. & Gal. $l$ & Gal. $b$ & $\begin{array}{c}\mathrm{GHB} T_{\mathrm{eff}} \\
E(B-V)=\mathrm{KIC}\end{array}$ & $\begin{array}{c}\mathrm{GHB} T_{\mathrm{eff}} \\
E(B-V)=0\end{array}$ & $\begin{array}{c}\Delta v \\
(\mu \mathrm{Hz})\end{array}$ & $\begin{array}{c}v_{\max } \\
(\mu \mathrm{Hz})\end{array}$ & $\begin{array}{l}\text { Star } \\
\text { Flags }\end{array}$ \\
\hline 10907196 & $\mathrm{~J} 18583782+4822494$ & 284.658 & 48.380 & 78.402 & 18.804 & $4969 \pm 79$ & $4808 \pm 74$ & $4.67 \pm 0.13$ & $44.12 \pm 0.96$ & KASC, PUBLIC \\
\hline 10962775 & $\mathrm{~J} 18582020+4824064$ & 284.584 & 48.402 & 78.405 & 18.857 & $5009 \pm 81$ & $4819 \pm 75$ & $4.11 \pm 0.09$ & $35.12 \pm 0.86$ & PUBLIC \\
\hline 11177749 & $\mathrm{~J} 18571019+4848067$ & 284.292 & 48.802 & 78.735 & 19.174 & $4769 \pm 105$ & $4612 \pm 99$ & $4.11 \pm 0.10$ & $34.18 \pm 0.79$ & KASC \\
\hline 11231549 & $\mathrm{~J} 18584464+4857075$ & 284.686 & 48.952 & 78.977 & 18.981 & $4728 \pm 80$ & $4574 \pm 75$ & $3.40 \pm 0.08$ & $30.25 \pm 0.69$ & KASC \\
\hline 11284798 & $\mathrm{~J} 18582108+4901359$ & 284.588 & 49.027 & 79.028 & 19.067 & $4349 \pm 70$ & $4201 \pm 65$ & $1.46 \pm 0.04$ & $9.50 \pm 0.25$ & KASC, LUMINOUS \\
\hline
\end{tabular}

(This table is available in its entirety in a machine-readable form in the online journal. A portion is shown here for guidance regarding its form and content.)

Table 4

Scale 1 Asteroseismic Results

\begin{tabular}{|c|c|c|c|c|c|c|}
\hline KIC ID & $\begin{array}{l}\text { ASPCAP } T_{\text {eff }} \\
(\text { Raw })(\mathrm{K})\end{array}$ & $\begin{array}{c}\text { ASPCAP }[\mathrm{M} / \mathrm{H}] \\
(\mathrm{Raw})\end{array}$ & $\begin{array}{c}\text { Mass } \\
M_{\odot}\end{array}$ & $\begin{array}{c}\text { Radius } \\
R_{\odot}\end{array}$ & $\log g$ & $\begin{array}{c}\rho \\
\text { (Solar Units) }\end{array}$ \\
\hline 10907196 & $4721 \pm 87$ & $-0.08 \pm 0.06$ & $1.47_{-0.19}^{+0.16}$ & $10.77_{-0.64}^{+0.42}$ & $2.543_{-0.011}^{+0.011}$ & $0.00119_{-0.00005}^{+0.00005}$ \\
\hline 10962775 & $4716 \pm 94$ & $-0.25 \pm 0.06$ & $1.21_{-0.12}^{+0.13}$ & $10.93_{-0.48}^{+0.47}$ & $2.444_{-0.012}^{+0.011}$ & $0.00093_{-0.00003}^{+0.00003}$ \\
\hline 11177749 & $4591 \pm 83$ & $0.03 \pm 0.05$ & $1.08_{-0.11}^{+0.12}$ & $10.51_{-0.45}^{+0.49}$ & $2.426_{-0.011}^{+0.011}$ & $0.00093_{-0.00003}^{+0.00003}$ \\
\hline 11231549 & $4451 \pm 86$ & $-0.06 \pm 0.06$ & $1.51_{-0.15}^{+0.17}$ & $13.31_{-0.56}^{+0.58}$ & $2.368_{-0.011}^{+0.011}$ & $0.00064_{-0.00002}^{+0.00002}$ \\
\hline 11284798 & $4126 \pm 85$ & $-0.04 \pm 0.06$ & $1.22_{-0.14}^{+0.17}$ & $21.76_{-1.05}^{+1.14}$ & $1.848_{-0.013}^{+0.013}$ & $0.00012_{-0.00001}^{+0.00001}$ \\
\hline
\end{tabular}

(This table is available in its entirety in a machine-readable form in the online journal. A portion is shown here for guidance regarding its form and content.)

Table 5

Scale 2 Asteroseismic Results

\begin{tabular}{|c|c|c|c|c|c|c|}
\hline KIC ID & $\begin{array}{c}\text { ASPCAP } T_{\text {eff }} \\
\text { (Corrected) }\end{array}$ & $\begin{array}{l}\text { ASPCAP }[\mathrm{M} / \mathrm{H}] \\
(\text { Corrected })\end{array}$ & Mass & Radius & $\log g$ & $\rho$ \\
\hline 10907196 & $4740 \pm 87$ & $-0.08 \pm 0.06$ & $1.50_{-0.20}^{+0.13}$ & $10.88_{-0.72}^{+0.33}$ & $2.543_{-0.011}^{+0.011}$ & $0.00119_{-0.00004}^{+0.00005}$ \\
\hline 10962775 & $4736 \pm 94$ & $-0.29 \pm 0.06$ & $1.21_{-0.12}^{+0.13}$ & $10.94_{-0.45}^{+0.48}$ & $2.444_{-0.012}^{+0.011}$ & $0.00093_{-0.00003}^{+0.00003}$ \\
\hline 11177749 & $4644 \pm 83$ & $0.06 \pm 0.05$ & $1.08_{-0.11}^{+0.13}$ & $10.54_{-0.46}^{+0.50}$ & $2.427_{-0.010}^{+0.011}$ & $0.00093_{-0.00003}^{+0.00003}$ \\
\hline 11231549 & $4541 \pm 86$ & $-0.03 \pm 0.06$ & $1.55_{-0.16}^{+0.17}$ & $13.46_{-0.58}^{+0.61}$ & $2.371_{-0.011}^{+0.011}$ & $0.00064_{-0.00002}^{+0.00002}$ \\
\hline 11284798 & $4283 \pm 85$ & $0.04 \pm 0.06$ & $1.29_{-0.15}^{+0.16}$ & $22.26_{-1.07}^{+1.12}$ & $1.854_{-0.012}^{+0.012}$ & $0.00012_{-0.00001}^{+0.00001}$ \\
\hline
\end{tabular}

(This table is available in its entirety in a machine-readable form in the online journal. A portion is shown here for guidance regarding its form and content.)

and through a grid-based search. In both cases, the distribution of the deviation between the true and the inferred properties is sharper for the grid-based method. Although differences between the distributions are not as large as in the case of mainsequence or subgiant stars, the difference, particularly in mass, is large enough that it is worth performing grid modeling for our sample to reduce our random uncertainties. As discussed above, grid modeling is more challenging in the $\log g$ regime where both the RGB and RC are present, and RC models and stars are not included in this comparison.

\section{THE APOKASC CATALOG AND ITS PROPERTIES}

\subsection{The APOKASC Catalog}

We present our data in three tables. Table 3 contains basic information on the stars, and our asteroseismic parameters for the raw and corrected temperature scale are given in Tables 4 and 5 . We begin in Table 3 with the information in common for both sets of spectroscopic inputs. We begin with star identifiers and positional information: the KIC (first column) and 2MASS (second column) IDs of the targets; right ascension (R.A.) and declination (decl.) (J2000), Galactic longitude $l$ and latitude $b$ (all in degrees) are presented in Columns 3-6. We present two different $T_{\text {eff }}$ measurements (in $\mathrm{K}$ ) estimates using the GHB09 methodology in Columns 7 and 8: one with the KIC extinction and the other with zero extinction. The uncertainties reflect random errors in the $J-K$ color used for the temperature estimates. This is distinct from the published effective temperatures presented in Pinsonneault et al. (2012), which used either SDSS filters or the Casagrande et al. (2010) IRFM temperatures from 2MASS colors. For each star that passed our internal consistency checks, we then provide the mean asteroseismic properties $\Delta v$ and $v_{\max }$ (in $\mu \mathrm{Hz}$ ) and their uncertainties in Columns 9 and 10. The final column includes the targeting flags used to select the stars for APOGEE observations. Stars included in GO proposal No. 40033 are labeled GO; stars targeted as public giants are PUBLIC (note that this includes almost all targets not newly observed for the GO program). A small number of asteroseismic "dwarfs" with long-cadence frequency measurements are labeled as SEIS DWARF.

Our detection efficiency was a function of surface gravity, which reflects physical and sampling effects that make obtaining reliable results more challenging for some targets than for others. The highest gravity stars had oscillation frequencies close to the Nyquist frequency, which can create model-dependent results. Low gravity stars have fewer modes and their interpretation is also more model dependent. The stars for which we did not obtain consistent results were clustered around these two 


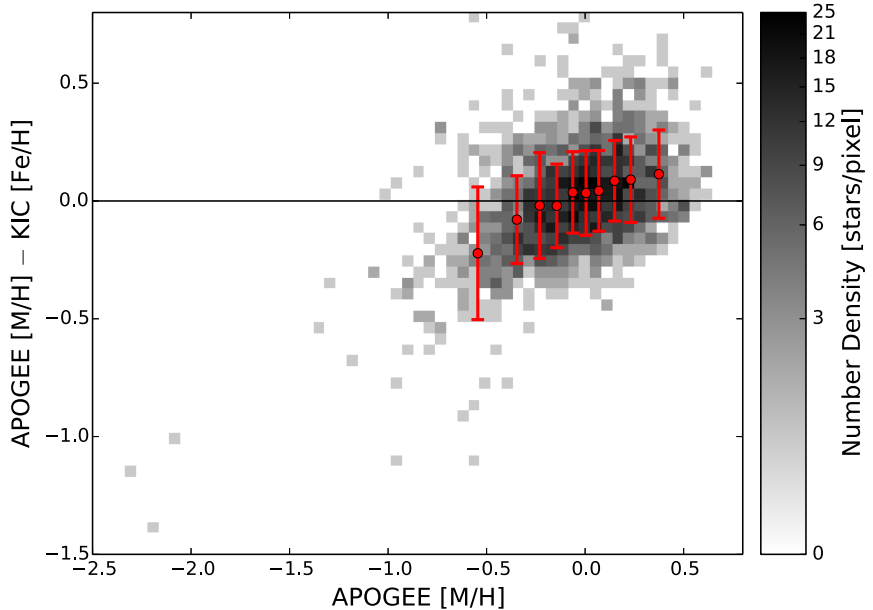

Figure 11. Logarithmic difference in metallicity between the APOKASC and KIC metallicities as a function of APOKASC metallicity. The points with error bars are the means and standard deviations of the data in 10 ranked cohorts of APOKASC metallicity. The data were divided into 60 bins in metallicity and metallicity difference, covering the range -2.5 to +0.8 and -1.5 to +0.8 dex, respectively, and the logarithmic color coding (specified on the right) indicates the number of targets with those properties. The correspondence between the $\mathrm{KIC}$ and spectroscopic results for giants is closer than that reported previously for dwarfs.

(A color version of this figure is available in the online journal.)

categories. For the analysis of the full DR12 catalog we intend to reanalyze and report results for all targets with detections, but we did not proceed with the full analysis of such objects in our initial run.

In Table 4 we present the asteroseismic properties derived from the uncorrected spectroscopic parameters; we refer to this set of measurement as Scale 1. Table 5 has an identical format except that it was derived using the corrected spectroscopic parameters, and we refer to these measurements as Scale 2. We start with the KIC ID, the input $T_{\text {eff }}$ in K, and $[\mathrm{M} / \mathrm{H}]$ (the logarithmic iron to hydrogen ratio relative to the Sun) for the relevant spectroscopic scale. We then present the mass (in solar units), radius (in solar units), surface gravity (log base 10 in cgs units), and mean density relative to that of the Sun; see Section 4 for the methodology used to derive the uncertainties. Although one could in principle construct mean densities and surface gravities from the masses and radii, we solved for these independently. The tabulated density and gravity values are close, but therefore not identical, to those which could be inferred from the separate $M$ and $R$.

\subsection{APOKASC and the KIC Compared}

A natural point of reference for our work are the stellar parameters in the KIC, which were based on photometry. Figure 11 displays the binned metallicity differences between our spectroscopic metallicities and those in the KIC inferred from photometry as a function of APOKASC metallicity. The average metallicities are surprisingly close, with a mean difference of 0.006 dex and a standard deviation of 0.22 dex. However, there are significant metallicity trends in the difference between the two, in the sense that the KIC metallicity scale is compressed relative to the APOGEE one. The effective temperature scale is also systematically cooler than that derived from the KIC, demonstrated in Figure 12. The offset $(-86 \mathrm{~K}$ on average) and dispersion ( $84 \mathrm{~K}$ ) are modest. The temperature scale differences are sensitive to the adopted extinction model

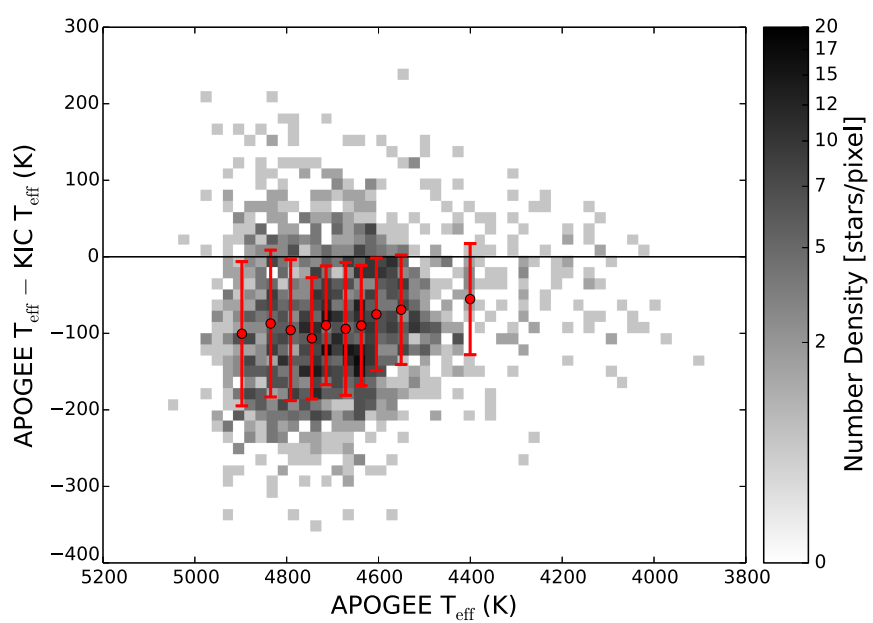

Figure 12. Difference in effective temperature between the APOKASC and KIC values as a function of APOKASC $T_{\text {eff }}(\mathrm{K})$. The points with error bars are the medians and median absolute deviations of the data in 10 ranked cohorts of APOKASC $T_{\text {eff }}$. The data were divided into 60 bins in $T_{\text {eff }}$ and $\Delta T_{\text {eff }}$, covering the range $3800-5200 \mathrm{~K}$ and -400 to $+300 \mathrm{~K}$, respectively, and the logarithmic color coding (specified on the right) indicates the number of targets with those properties. The major difference between the two systems is a zero-point offset. (A color version of this figure is available in the online journal.)

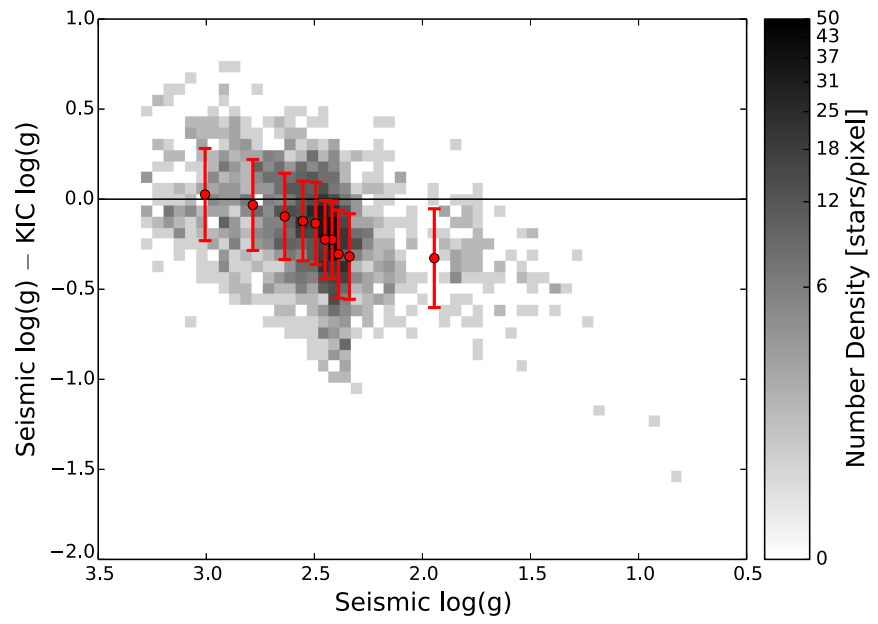

Figure 13. Logarithmic difference in surface gravity $\log g$ between the APOKASC and KIC values as a function of APOKASC $\log g$. The points with error bars are the medians and median absolute deviations of the data in 10 ranked cohorts of APOKASC $\log g$. The data were divided into 60 bins in $\log g$ and delta $\log g$, covering the range $0.5-3.5$ and -2.0 to +1.0 , respectively, and the logarithmic color coding (specified on the right) indicates the number of targets with those properties.

(A color version of this figure is available in the online journal.)

and to the color-temperature calibration, an issue that we return to below. Finally, the differences in surface gravity are illustrated in Figure 13. The KIC surface gravities have a large scatter of 0.265 dex relative to our asteroseismic values, with a mean difference of -0.175 dex.

Motivated by these results, we derived fitting functions mapping the KIC parameters onto the spectroscopic system. We emphasize that these results are calibrated for red giants only, and different relations may well apply for dwarfs or subgiants. The fits between the KIC parameters and the corrected ASPCAP $[\mathrm{M} / \mathrm{H}], T_{\text {eff }}$, and asteroseismic $\log g$ are shown in Figures 14-16, respectively. Our fitting formulae correcting the KIC values to the spectroscopic scale are

$$
[\mathrm{M} / \mathrm{H}]_{\mathrm{ASPCAP}}=0.72 *[\mathrm{Fe} / \mathrm{H}]_{\mathrm{KIC}}+0.03,
$$



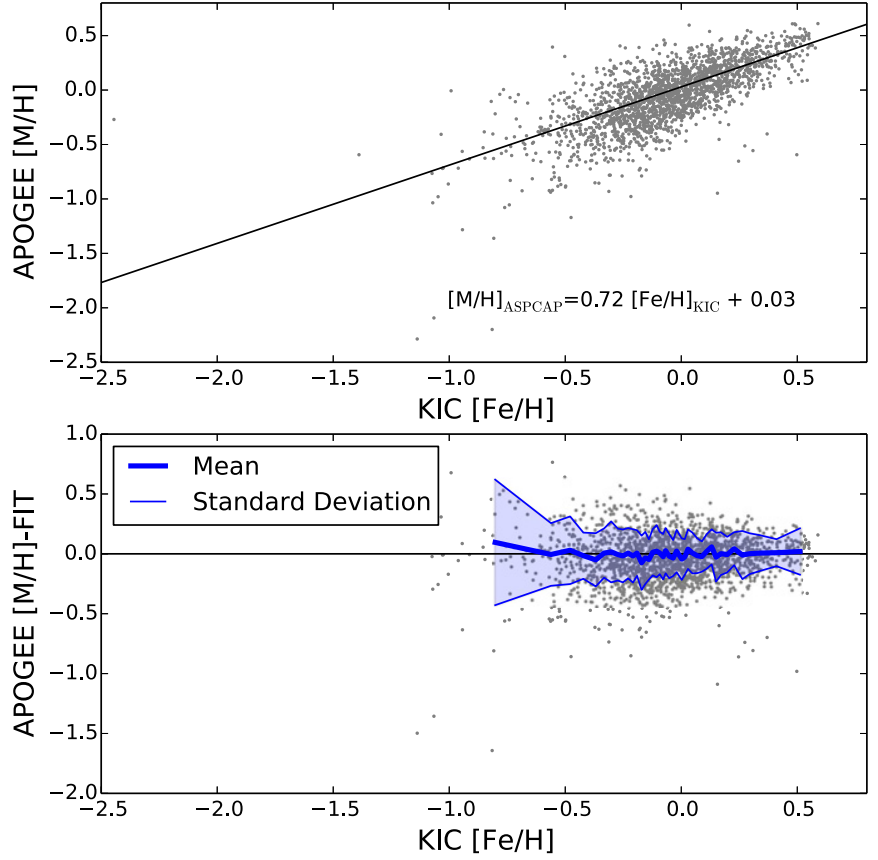

Figure 14. Results of a linear fit between the KIC- and ASPCAP-corrected metallicities. A strong metallicity trend is seen in the residuals, indicating a lower precision for photometric metallicity estimates in metal-poor stars.

(A color version of this figure is available in the online journal.)
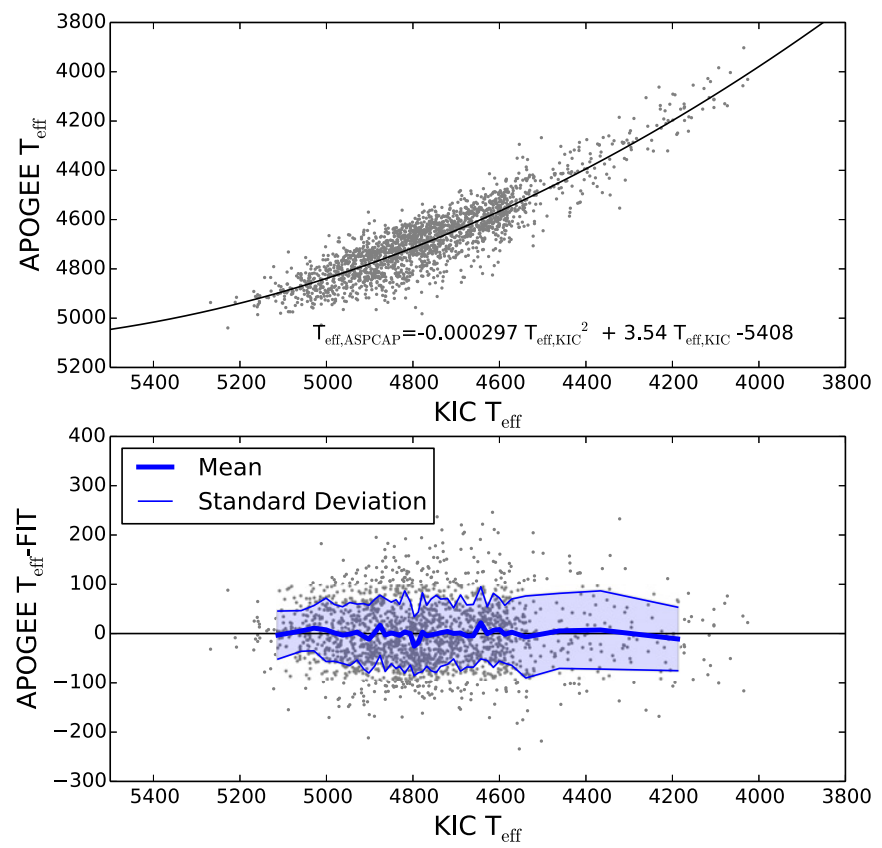

Figure 15. Results of a quadratic fit between the KIC- and ASPCAPcorrected $T_{\text {eff }}$ values. There are only weak trends in uncertainties with effective temperature. Hot stars, which are mostly in the secondary clump in our sample, are largely responsible for the curvature in the fitting function.

(A color version of this figure is available in the online journal.)

$$
\begin{aligned}
& T_{\text {eff,ASPCAP }}=0.000297 * T_{\text {eff, KIC }}^{2}+3.54 * T_{\text {eff, } \mathrm{KIC}}-5408, \\
& \log g_{\text {ASPCAP }}=0.41 * \log g_{\text {KIC }}+0.00054 * T_{\text {eff, }, \mathrm{KIC}}-1.13 .
\end{aligned}
$$

The top panel of each figure shows the best-fit line compared to the data, with the function in the lower right. The dark and light blues curves are the weighted mean and magnitude of the
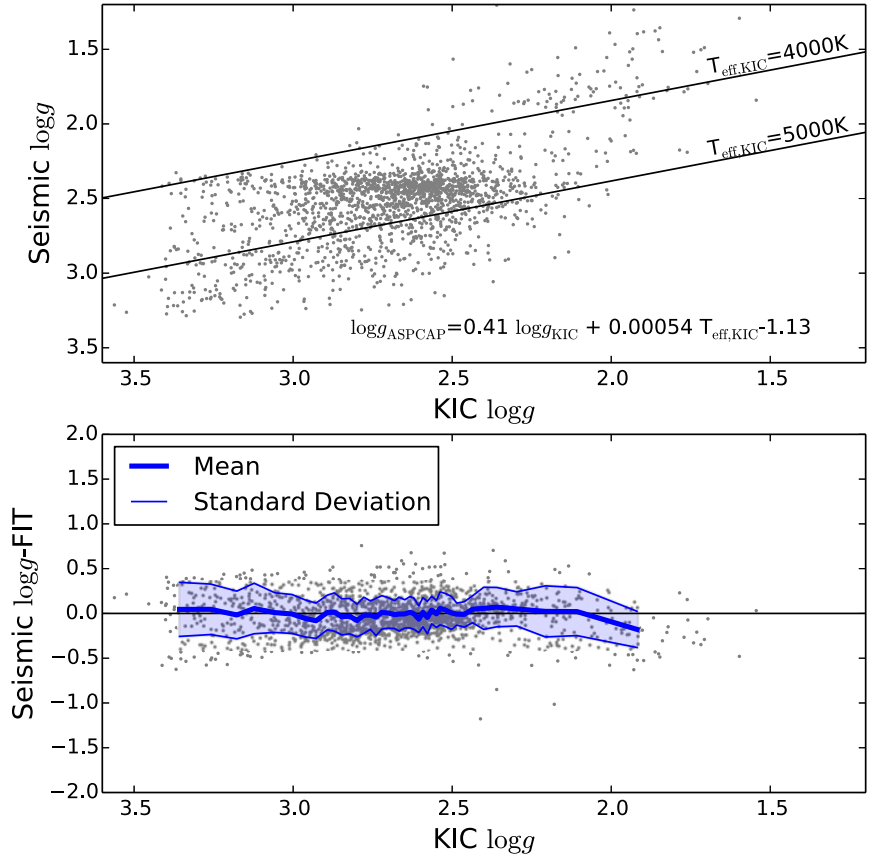

Figure 16. Results of a bilinear fit between the KIC- and ASPCAP-corrected $T_{\text {eff }}$ values. Our best-fit relations for cool and hot giants correspond to the two lines on the figure.

(A color version of this figure is available in the online journal.)

standard deviation, respectively (computed by dividing the data into 40 bins containing equal numbers of stars). A linear function was a good fit for Equation (3). Curvature in the differences at high temperatures made a quadratic function a better fit than a linear one for Equation (4), and correlations between the gravity offsets, $T_{\text {eff }}$, and $\log g$ drove our choice of functional form for Equation (5). The scatter around the $\mathrm{KIC}[\mathrm{Fe} / \mathrm{H}]$-corrected ASPCAP $[\mathrm{M} / \mathrm{H}]$ relationship increases toward lower metallicity stars; this result is not surprising for a photometric system, which tends to lose sensitivity for more metal-poor objects. Dong et al. (2014) performed a related exercise comparing LAMOST and KIC metallicities for dwarfs. In their case, they reported a comparable dispersion in the relative metallicities but a different functional form for the fit. This discrepancy is likely to be tied to the difference between the photometric calibrations for giants and dwarfs. Similar effects are also seen for $T_{\text {eff }}$ and $\log g$, in the sense that the dwarf and giant offsets between the KIC and comparison samples are not the same.

Our final external check is against a sample of results from optical spectroscopy obtained by Thygesen et al. (2012). Those authors compared their spectroscopic results against both asteroseismic surface gravities and the KIC. That paper reported a close correspondence between the mean KIC and spectroscopic properties, with spectroscopic minus KIC differences $\Delta T_{\text {eff }}$, $\Delta \log g$, and $\Delta[\mathrm{Fe} / \mathrm{H}]$ of $3 \mathrm{~K}(\sigma=105 \mathrm{~K}),-0.003 \operatorname{dex}(\sigma=$ $0.67 \mathrm{dex})$, and $0.003 \mathrm{dex}(\sigma=0.50 \mathrm{dex})$, respectively. Relative to the asteroseismic surface gravities, Thygesen et al. (2012) reported a smaller offset than the one that we obtain $(-0.05 \mathrm{dex})$ with a slightly larger scatter (0.30 dex). Further insight can be obtained from the stars in common between the two samples (Figure 17). The ASPCAP and optical spectroscopy results are in good agreement in both metallicity and surface gravity, and disagree only in effective temperature (at the $86 \mathrm{~K} \mathrm{level).} \mathrm{The}$ close agreement between our results and those of Thygesen et al. (2012) for stars in common between the two samples indicates 

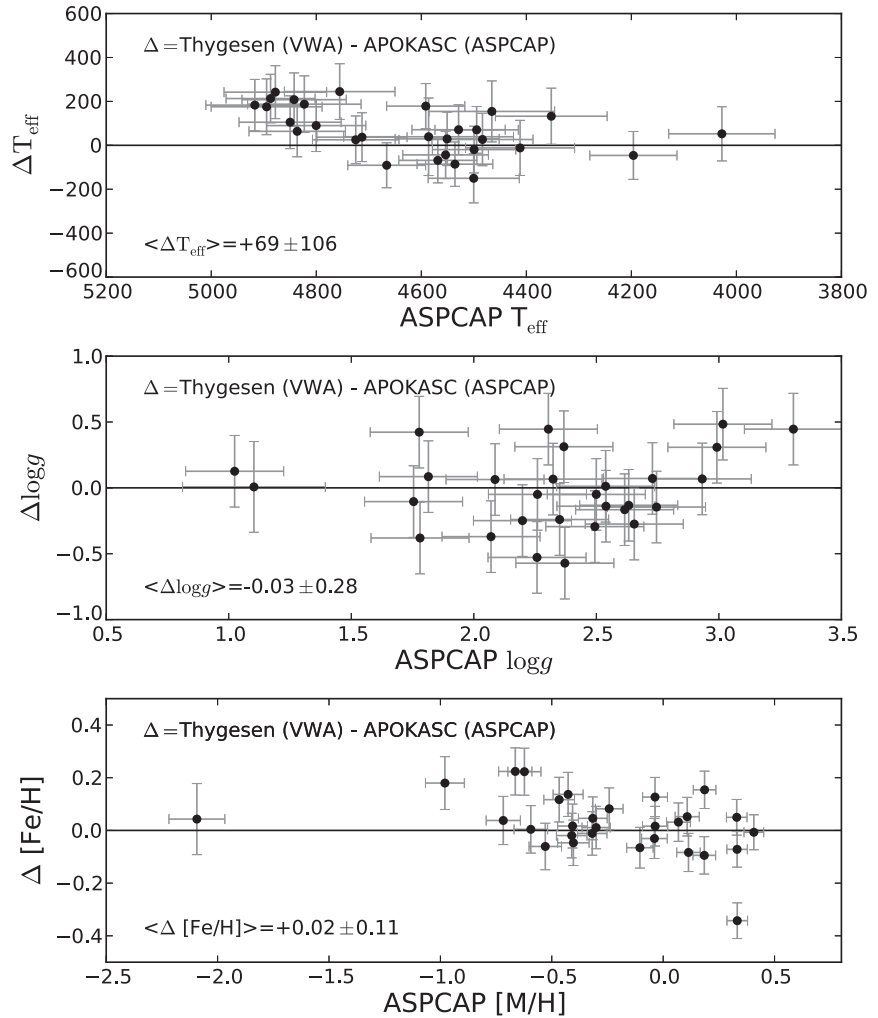

Figure 17. Temperature, surface gravity, and $[\mathrm{Fe} / \mathrm{H}]$ differences, respectively, for stars in common between APOGEE and Thygesen et al. (2012) APOGEE internal errors are used. Thygesen individual error bars were not directly reported, so they were inferred to be half (in quadrature) of the differences between their optical results and external literature comparisons. The derived dispersions between optical and IR spectra are indicated on the figures and are consistent with this uncertainty measurement.

that there is no global $\log g$ offset between the two spectroscopic methods. Because our sample is large and the uncertainties in the asteroseismic gravities are small, our mean offset of -0.175 dex between the KIC and asteroseismic scales is highly statistically significant, which differs from the -0.05 dex offset reported by Thygesen et al. (2012). Since the two methods agree in their results for the stars in common, the origin of this difference is likely to be in sampling effects (e.g., our sample includes stars where the asteroseismic and KIC surface gravities simply disagree more on average than a typical star in the smaller Thygesen sample). The zero-point offset between the corrected spectroscopic temperatures and those of Thygesen is comparable in magnitude and sign with that inferred relative to the photometric GHB09 scale. The temperature zero-point offsets, however, appear to have minimal impact on the metallicities or surface gravities.

\subsection{Global Properties of the APOKASC Sample}

The combination of asteroseismic and spectroscopic data adds new dimensions to traditional stellar population studies. As an example, we present three different $\mathrm{H}-\mathrm{R}$ diagrams for the stars in our sample in Figure 18. The left panel reflects the photometric parameters inferred from the KIC. With the addition of spectroscopic data distinct features begin to emerge, in particular a prominent RC. However, with the addition of asteroseismic surface gravities, fine structure can clearly be seen in a field population without parallax data. Both the RC and the secondary RC can be clearly distinguished, and the RGB bump (typically detected only in star clusters) is clearly visible.

We can also examine trends in mass at fixed metallicity (illustrated in Figure 19) and in metallicity at fixed mass (illustrated in Figure 20). The overall trends predicted by stellar models are clearly seen, with metal-poor stars being systematically hotter at fixed $\log g$ and mass than metal-rich ones. Similarly, higher mass stars, as expected, are systematically hotter at fixed metallicity than lower mass stars. The agreement between theory and data degrades for the lowest mass stars, which could imply a mass-dependent shift in the locus of the giant branch. However, this may simply be a stellar population effect: the limited age of the disk places a hard lower bound on the true mass of evolved red giants. Stars with formal mass estimates at or close to this value are likely to be higher mass stars scattered to a low apparent mass by errors in their data, and they will thus appear offset relative to expectations. For the YREC models there is good agreement at low metallicity but there
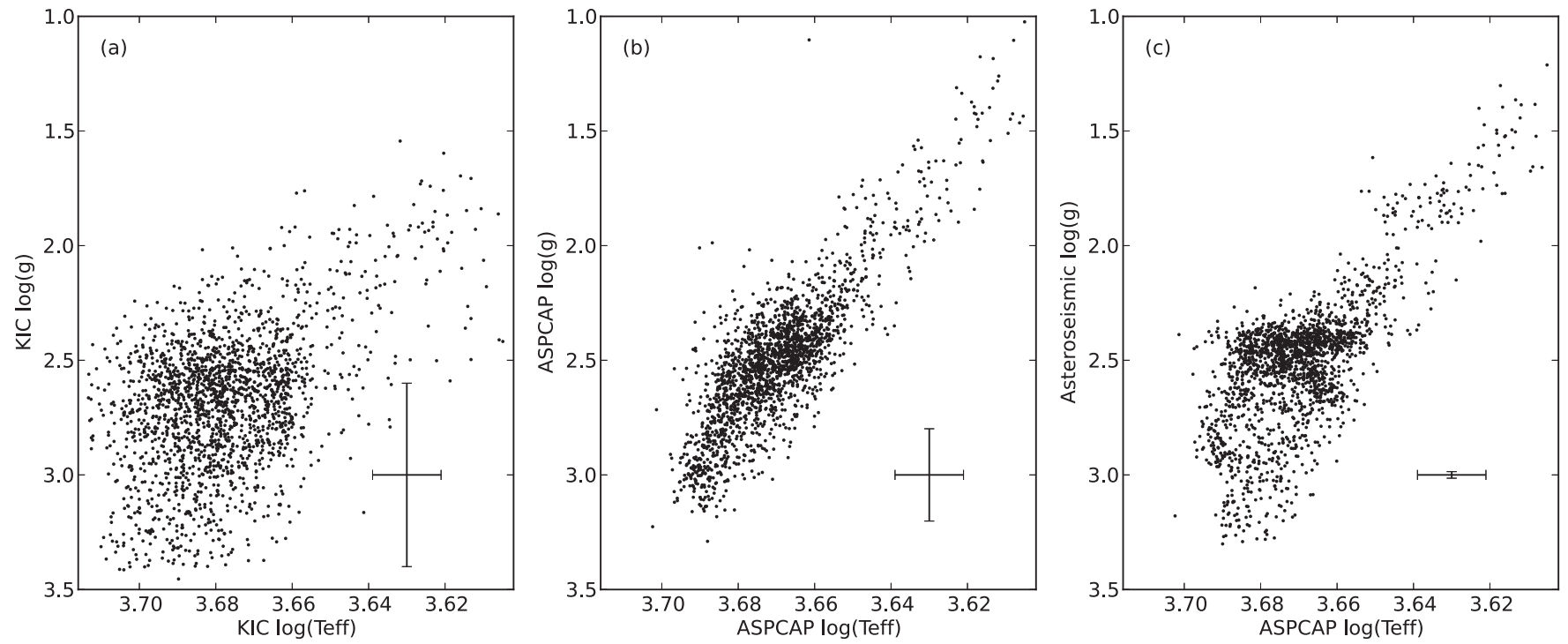

Figure 18. Our catalog stars in $\log g-T_{\text {eff }}$ space for three different methods. The original, purely photometric system for the KIC is in the left panel. A purely spectroscopic system, corresponding to the parameters released in DR10, is in the center panel. Our published parameters, which differ by the addition of asteroseismic surface gravities, produces the right panel. The stellar populations in the field snap into focus as we provide additional information. 

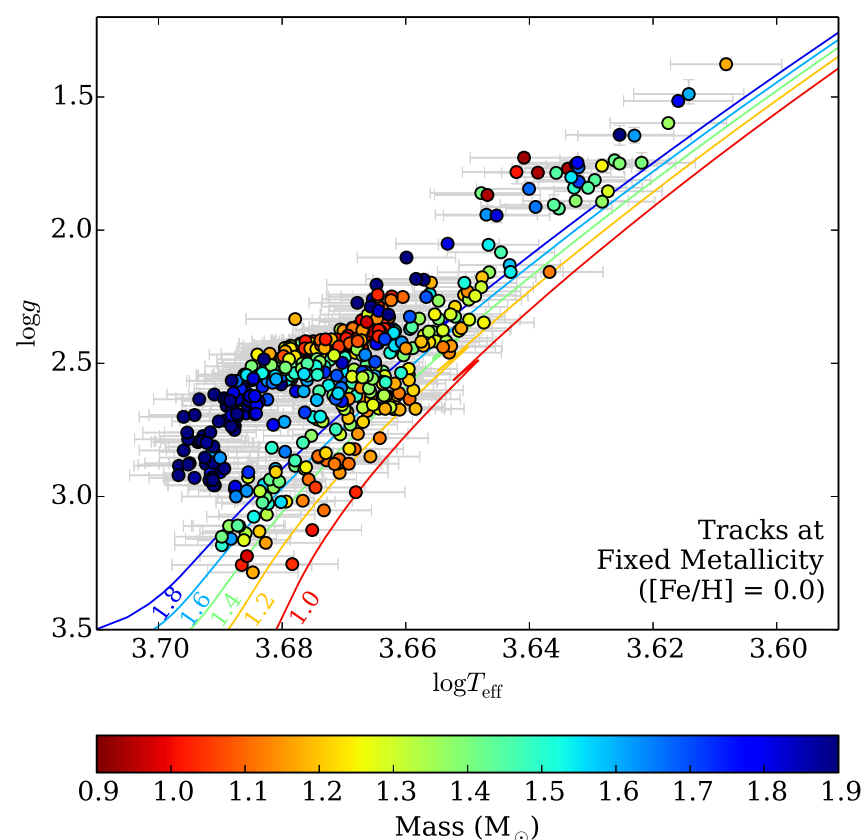
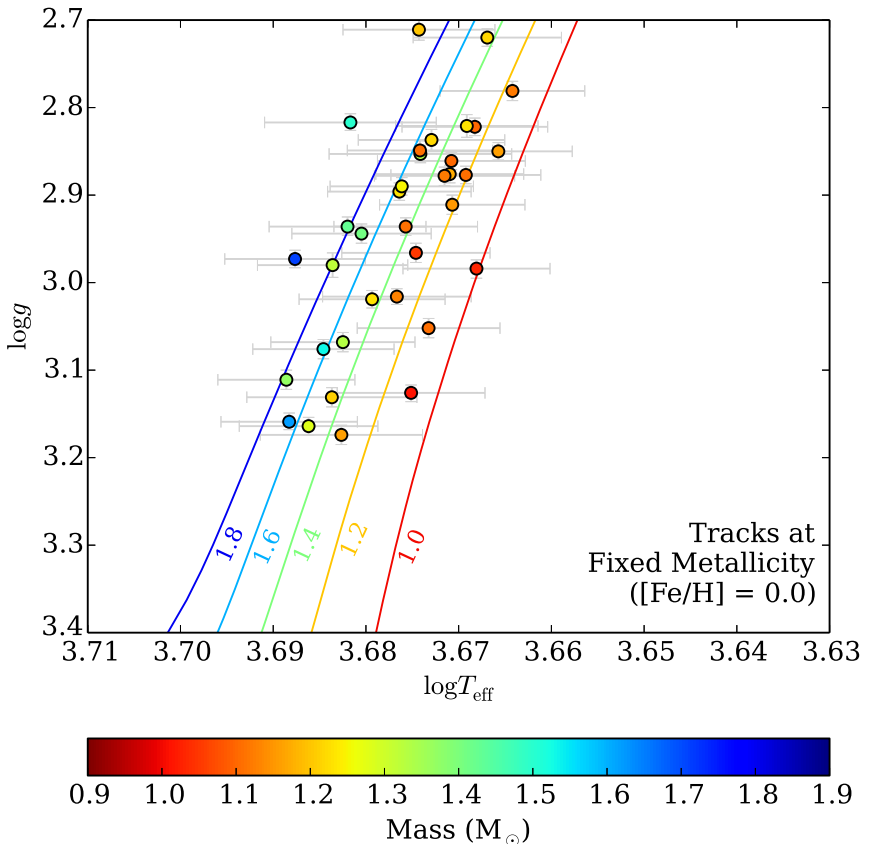

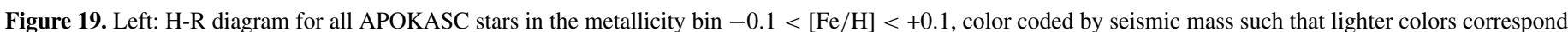

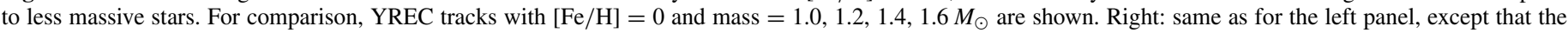

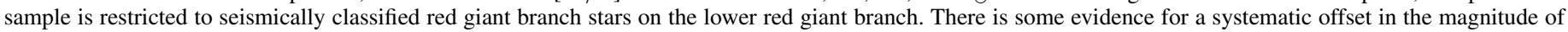
the mass trend for the lowest mass targets, but this could reflect the finite age of the disk; see text.

(A color version of this figure is available in the online journal.)
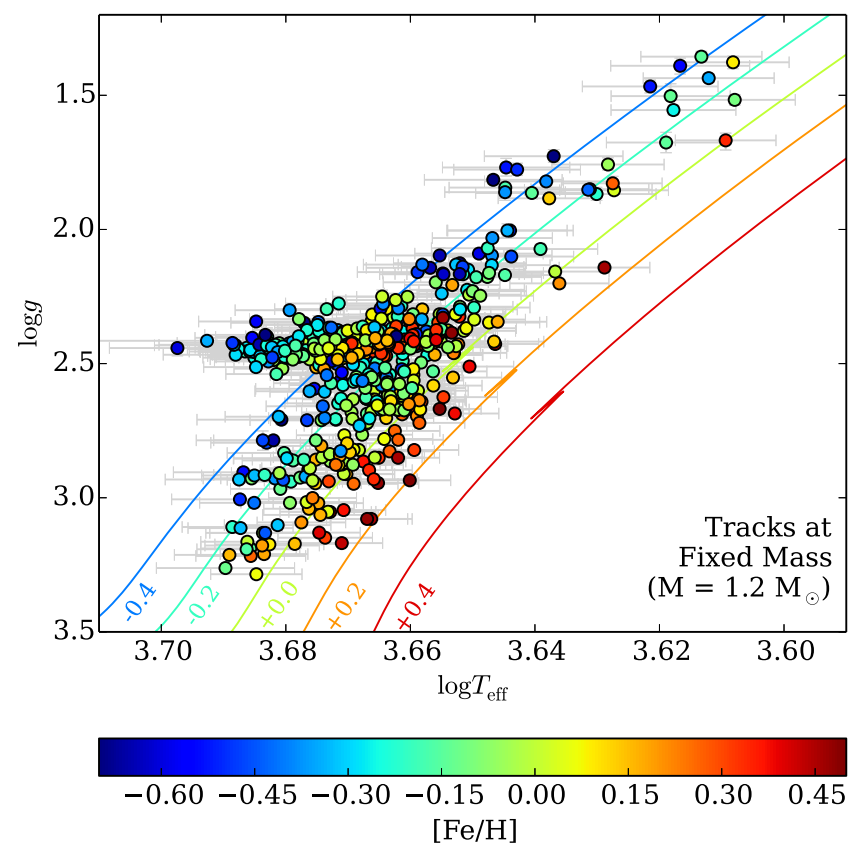
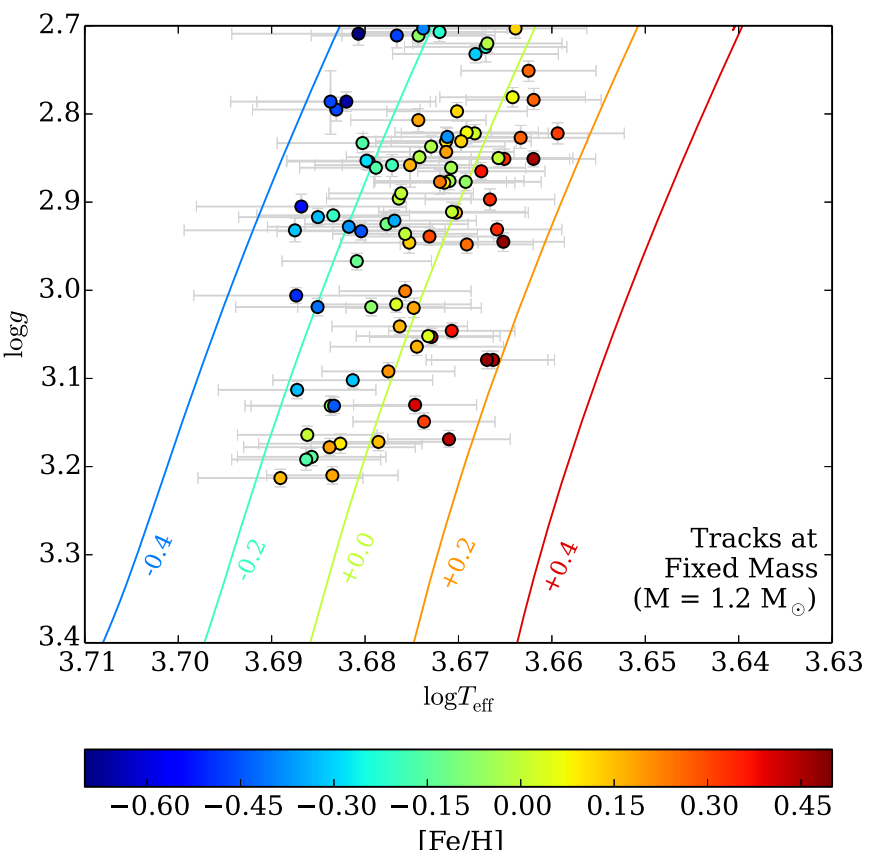

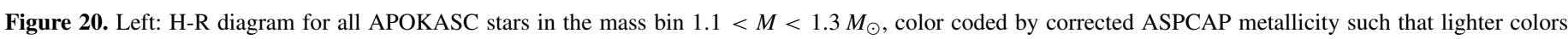

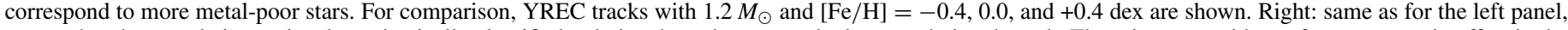

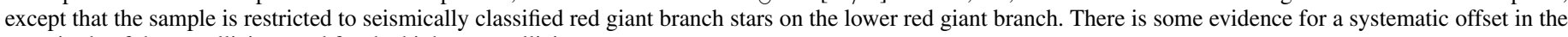
magnitude of the metallicity trend for the highest metallicity targets.

(A color version of this figure is available in the online journal.)

appears to be an offset in the temperature locus between the metal-rich track and the metal-rich data. This result is more robust than that in the mass plane and is tentative evidence for a metallicity-dependent offset between the expected and observed H-R diagram position at fixed mass. Thygesen et al.
(2012) reported evidence for a metallicity (but not mass) dependent shift in the H-R diagram position of stars with asteroseismic masses and gravities relative to theoretical expectations. Our results are consistent with those that they derived from their sample. 


\section{CONCLUSIONS, CAUTIONS, AND FUTURE PROSPECTS}

Asteroseismology and high-resolution spectroscopy are powerful and complementary astrophysical tools. In this paper we present the APOKASC catalog, which is the first large and homogeneous catalog containing data from both high-resolution spectroscopy and asteroseismology. This effort provides new tools for studying stellar populations and testing the theories of both stellar atmospheres and stellar interiors. We now have a data set measured in the natural co-ordinate for stellar evolution calculations, namely, mass. This permits direct tests of stellar evolution tracks using isolated field stars, as opposed to relying on membership in well-studied binary systems or star clusters. Asteroseismic data is particularly precise and accurate for surface gravities, which is a stringent test of model atmospheres theory. Our combined data delineate stellar populations in the field with a precision usually associated with star clusters, and asteroseismic evolutionary state diagnostics confirm the assignments (such as RC, secondary RC, or RGB) that would have been expected from the H-R diagram positions of the stars. Differential mass trends (at fixed metallicity) and metallicity trends (at fixed mass) are in the expected sense, which is a powerful confirmation of expectations from stellar interiors theory. At a deeper level, interesting trends in the differences between theory and data for both atmospheres and interiors models are clearly present and worthy of further exploration.

Typical uncertainties in our catalog are of the order of $80 \mathrm{~K}$ in $T_{\text {eff }}, 0.06 \operatorname{dex}$ in $[\mathrm{M} / \mathrm{H}], 0.014 \mathrm{dex}$ in $\log g$, and $12 \%$ and 5\% in mass and radius, respectively. There are additional systematic error sources that could be important and which should be explored. For example, a zero-point shift in the $T_{\text {eff }}$ scale is plausible given the differences between photometric and spectroscopic inferences for our targets, which would induce correlated changes in the mass and radius values. An improved extinction map for the Kepler fields would permit a more precisely anchoring of the absolute scale and constrain this effect. Our metallicities show no evidence of systematic differences from targets measured with optical spectroscopy. We have derived mappings of KIC properties onto the system defined in this paper, and find a reasonable correspondence between the KIC and spectroscopic metallicities for giants. The accurate stellar properties and distances available in the APOKASC sample in the Kepler field are also useful for calibrating stellar-population tracers, such that the power of the APOKASC sample can be leveraged to the large Galactic volume covered by the full APOGEE survey. As an example of this potential, Bovy et al. (2014) recently developed a new method for selecting RC stars from spectro-photometric data that is calibrated using the precise seismic $\log g$ and evolutionary-state classifications in the APOKASC catalog. The distances to stars in the resulting APOGEE-RC catalog are accurate to $\sim 5 \%$, allowing for a detailed mapping of the structure of the Galactic disk. The seismic data are also useful to check for systematic errors in the RC distance scale, which is calibrated using Hipparcos parallaxes: the direct seismic distances for 593 $\mathrm{RC}$ stars in common between the APOGEE-RC catalog and the Rodrigues et al. (2014) distance catalog agree to better than $1 \%$.

At the same time, there are important factors that should be accounted for when interpreting and using the data. There are significant sample selection effects, imposed by the target selection process for both Kepler and APOGEE. These selection effects are not simple ones (for example, magnitude or color cuts), and they reflect the fact that the asteroseismic data was obtained from a survey designed for a very different purpose (detecting extrasolar planets). Direct stellar populations inferences from our targets therefore require careful population modeling, which is outside the scope of our paper.

The availability of stellar masses is especially valuable for red giant stars, as the natural process of stellar evolution channels stars of very different masses and ages into similar locations on the H-R diagram. The two major methods for inferring stellar masses also have significant limitations when applied to evolved stars, making an additional mass diagnostic even more important. Direct mass measurements from binary stars are uncommon for physically large red giant stars. Indirect mass estimates for red giants in star clusters are subject to significant systematic uncertainties in cluster ages, distances, extinctions, and the mapping from turnoff properties to stellar mass. By the same token, however, our asteroseismic masses have only a limited set of calibrators. Our radii are based on the same scaling relations used in Silva Aguirre et al. (2012) and Huber et al. (2012), which reported good agreement with independent tests using stars with measured parallaxes and interferometric angular size measurements, particularly at solar metallicity. However, data at higher and lower metallicity are more limited, and there could be metallicity-dependent offsets in the mass scale. Epstein et al. (2014), for example, found that the masses derived from scaling relations for metal-poor stars were consistently higher than those expected from other astrophysical constraints. Miglio et al. (2012) found evolutionary-state-dependent differences between the radii inferred for RC and RGB stars in the metalrich open cluster NGC 6791. As a result, there could be systematic trends in our inferred masses and radii not captured in our analysis. There are systematic differences between the spectroscopic and asteroseismic surface gravities that appear to be a function of evolutionary state as well. Calibration of the asteroseismic masses and radii, and understanding the origin of the trends that have been identified, is ongoing.

Work is in progress on the next combined data set which will feature much larger numbers of targets and considerably broader phase space coverage in metallicity and surface gravity. Our asteroseismic uncertainties reflect a combination of systematic and random error sources, and work is in progress to assess both separately. We also adopted separate external calibrators for each of our spectroscopic parameters; in the next version of the catalog we will explore the consequences of iterating between the asteroseismic and spectroscopic parameters and exploring the impact of adopting an asteroseismic gravity prior for the spectroscopic solution. In the current paper we employed a conservative concordance criterion for our asteroseismic sample (see Section 4.1), reporting results only in cases where different analysis methods applied to the same data set yielded similar asteroseismic parameters. This approach had the net effect of removing most of the highest and lowest gravity targets from our sample, as their data are the most subject to systematic offsets. Including these in the next catalog, which will substantially increase our dynamic range in surface gravity, is another priority. We also plan to take full advantage of the improvements in the ASPCAP spectroscopic pipeline.

Funding for SDSS-III has been provided by the Alfred P. Sloan Foundation, the Participating Institutions, the National Science Foundation, and the U.S. Department of Energy Office of Science. The SDSS-III Web site is http://www.sdss3.org/. SDSS-III is managed by the Astrophysical Research Consortium for the Participating Institutions of the SDSS-III 
Collaboration including the University of Arizona, the Brazilian Participation Group, Brookhaven National Laboratory, Carnegie Mellon University, University of Florida, the French Participation Group, the German Participation Group, Harvard University, the Instituto de Astrofisica de Canarias, the Michigan State/ Notre Dame/JINA Participation Group, Johns Hopkins University, Lawrence Berkeley National Laboratory, Max Planck Institute for Astrophysics, Max Planck Institute for Extraterrestrial Physics, New Mexico State University, New York University, Ohio State University, Pennsylvania State University, University of Portsmouth, Princeton University, the Spanish Participation Group, University of Tokyo, University of Utah, Vanderbilt University, University of Virginia, University of Washington, and Yale University.

C.E., J.J., M.P., and J.T. acknowledge support from NSF grant AST-1211673. D.H. acknowledges support by an appointment to the NASA Postdoctoral Program at Ames Research Center administered by Oak Ridge Associated Universities, and NASA grant NNX14AB92G issued through the Kepler Participating Scientist Program. D.S. acknowledges support from the Australian Research Council. S.B. acknowledges partial support from NSF grant AST-1105930 and NASA grant NNX13AE70G. Funding for the Stellar Astrophysics Center is provided by the Danish National Research Foundation (Grant agreement No. DNRF106). V.S.A. was supported by the European Research Council (grant agreement No. 267864). S.M. acknowledges support from the NASA grant NNX12AE17G. S.H. has received funding from the European Research Council under the European Community's Seventh Framework Programme (FP7/ 2007-2013)/ERC grant agreement No. 338251 (StellarAges) and by Deutsche Forschungsgemeinschaft (DFG) under grant SFB 963/1 "Astrophysical flow instabilities and turbulence." W.J.C., Y.E., and A.M. acknowledge support from the UK Science and Technology Facilities Council (STFC). D.A. acknowledges support provided by the National Research Foundation of Korea to the Center for Galaxy Evolution Research (No. 2010-0027910). A.S. is partially supported by the MICINN grant AYA2011-24704 and by the ESF EUROCORES Program EuroGENESIS (MICINN grant EUI2009-04170). T.C.B. acknowledges partial support for this work by grant PHY 0822648: Physics Frontiers Center/Joint Institute for Nuclear Astrophysics (JINA), awarded by the U.S. National Science Foundation. V.V.S. acknowledges support from NSF grant AST11-09888. D.A.G.H. and O.Z. acknowledge support provided by the Spanish Ministry of Economy and Competitiveness under grant AYA-2011-27754. We thank an anonymous referee for comments that improved the paper.

\section{REFERENCES}

Ahn, C. P., Alexandroff, R., Allende Prieto, C., et al. 2014, ApJS, 211, 17 Asplund, M. 2005, ARA\&A, 43, 481

Basri, G., Walkowicz, L. M., Batalha, N. M., et al. 2011, AJ, 141, 20

Basu, S., Chaplin, W. J., \& Elsworth, Y. 2010, ApJ, 710, 1596

Basu, S., Grundahl, F., Stello, D., et al. 2011, ApJL, 729, L10

Basu, S., Verner, G. A., Chaplin, W. J., \& Elsworth, Y. 2012, ApJ, 746, 76

Batalha, N. M., Borucki, W. J., Koch, D. G., et al. 2010, ApJL, 713, L109

Beck, P. G., Montalban, J., Kallinger, T., et al. 2012, Natur, 481, 55 Bedding, T. R., Huber, D., Stello, D., et al. 2010, ApJL, 713, L176 Bedding, T. R., Mosser, B., Huber, D., et al. 2011, Natur, 471, 608 Belkacem, K., Goupil, M. J., Dupret, M. A., et al. 2011, A\&A, 530, 142 Bergemann, M., Ruchti, G., Serenelli, A., et al. 2014, A\&A, 565, 89 Bildsten, L., Paxton, B., Moore, K., \& Macias, P. J. 2012, ApJL, 744, L6 Binney, J., Burnett, B., Kordopatis, G., et al. 2014, MNRAS, 439, 1231 Bovy, J., Nidever, D. L., Rix, H.-W., et al. 2014, ApJ, 790, 127
Bovy, J., Rix, H.-W., Liu, C., et al. 2012, ApJ, 753, 148

Brown, T. M., Gilliland, R. L., Noyes, R. W., \& Ramsey, L. W. 1991, ApJ, 368,599

Brown, T. M., Latham, D. W., Everett, M. E., \& Esquerdo, G. A. 2011, AJ, 142,112

Canterna, R. 1976, AJ, 81, 228

Casagrande, L., Ramirez, I., Melendez, J., Bessel, M., \& Asplund, M. 2010, A\&A, 512, 54

Casagrande, L., Silva Aguirre, V., Stello, D., et al. 2014, ApJ, 787, 110

Chaplin, W. J., Houdek, G., Appourchaux, T., et al. 2008, A\&A, 485, 813

Chaplin, W. J., Kjeldsen, H., Christensen-Dalsgaard, J., et al. 2011, Sci, 332,213

Chaplin, W. J., Kjeldsen, H., Christensen-Dalsgaard, J., et al. 2014, ApJS, 210, 1

Christensen-Dalsgaard, J. 2008, AP\&SS, 316, 13

Christensen-Dalsgaard, J. 1993, in ASP Conf. Ser. 42, Proc. GONG 1992, Seismic Investigation of the Sun and Stars, ed. T. M. Brown (San Francisco, CA: ASP), 347

Creevey, O. L., Doğan, G., Frasca, A., et al. 2012, A\& A, 537, 111

da Silva, L., Girardi, L., Pasquini, L., et al. 2006, A\&A, 458, 609

Deheuvels, S., García, R. A., Chaplin, W. J., et al. 2012, ApJ, 756, 19

Demarque, P., Guenther, D. B., Li, L. H., Mazumdar, A., \& Straka, C. W. 2008, Ap\&SS, 316, 31

Demarque, P., Woo, J.-H., Kim, Y.-C., \& Yi, S. K. 2004, ApJS, 155, 667

De Ridder, J., Barban, C., Baudin, F., et al. 2009, Natur, 459, 398

Dong, S., Zheng, Z., Zhu, Z., et al. 2014, ApJL, 789, L3

Dotter, A., Chaboyer, B., Jevremovic, D., et al. 2008, ApJS, 178, 89

Eisenstein, D. J., Weinberg, D. H., Agol, E., et al. 2011, AJ, 142, 72

Epstein, C. R., Elsworth, Y. P., Johnson, J. A., et al. 2014, ApJL, 785, L28

Freeman, K. C. 2011, in EAS Publication Series, Vol. 45, GAIA: At the Frontiers of Astrometry, ed. C. Turon, F. Meynadier, \& F. Arenou (Cambridge: Cambridge Univ. Press), 213

Freeman, K. C. 2012, in ASP Conf. Ser. 458, Proceedings of the Conference held 2011 May 16-20 in Shuzenji, Japan, Galactic Archaeology: Near-Field Cosmology and the Formation of the Milky Way, ed. W. Aoki, M. Ishigaki, T. Suda, T. Tsujimoto, \& N. Arimoto (San Francisco, CA: ASP), 393

Fukugita, M., Ichikawa, T., Gunn, J. E., et al. 1996, AJ, 111, 1748

Gai, N., Basu, S., Chaplin, W. J., \& Elsworth, Y. 2011, ApJ, 730, 63

García, R. A., Hekker, S., Stello, D., et al. 2011, MNRAS, 414, 6

García, R. A., Pérez Hernández, F., Benomar, O., et al. 2014a, A\&A, 563, 84

García, R. A., Ceillier, T., Salabert, D., et al. 2014b, A\&A, in press (arXiv:1403.7155)

Geisler, D., Claria, J. J., \& Minniti, D. 1991, AJ, 102, 1836

Gilmore, G., Randich, S., Asplund, M., et al. 2012, Msngr, 147, 25

Girardi, L., Bressan, A., Bertelli, G., \& Chiosi, C. 2000, A\&AS, 141, 371

Girardi, L., Groenewegen, M. A. T., Hatziminaoglou, E., \& da Costa, L. 2005, A\&AS, 436, 895

González-Hernández, J. I., \& Bonifacio, P. 2009, A\&A, 497, 497

Gunn, J. E., Siegmund, W. A., \& Al, E. J. M. E. 2006, AJ, 131, 87

Hayden, M. R., Holtzman, J. A., Bovy, J., et al. 2014, AJ, 147, 116

Hekker, S., Broomhall, A.-M., Chaplin, W. J., et al. 2010, MNRAS, 402, 2049

Hekker, S., Elsworth, Y., De Ridder, J., et al. 2011a, A\&A, 525, 131

Hekker, S., Elsworth, Y., Mosser, B., et al. 2012, A\&A, 544, A90

Hekker, S., Elsworth, Y., Mosser, B., et al. 2013, A\&A, 556, 59

Hekker, S., Gillliland, R. L., Elsworth, Y., et al. 2011b, MNRAS, 414, 2594

Huber, D., Bedding, T. R., Stello, D., et al. 2010, ApJ, 723, 1607

Huber, D., Bedding, T. R., Stello, D., et al. 2011, ApJ, 743, 143

Huber, D., Chaplin, W. J., Christensen-Dalsgaard, J., et al. 2013, ApJ, 767, 127

Huber, D., Ireland, M. J., Bedding, T. R., et al. 2012, ApJ, 760, 32

Huber, D., Silva Aguirre, V., Matthews, J., et al. 2014, ApJS, 211, 2

Huber, D., Stello, D., Bedding, T. R., et al. 2009, CoAst, 160, 74

Jenkins, J. M., Caldwell, D. A., Chandrasekaran, H., et al. 2010, ApJL, 713, L120

Kallinger, T., Mosser, B., Hekker, S., et al. 2010, A\&A, 522, 1

Kjeldsen, H., \& Bedding, T. R. 1995, A\&A, 293, 87

Kordopatis, G., Gilmore, G., Steinmetz, M., et al. 2013, AJ, 146, 134

Lai, D. K., Rockosi, C. M., Bolte, M., et al. 2009, ApJL, 697, L63

Lee, Y.-S, Beers, T. C., Sirarani, T., et al. 2008, AJ, 136, 2050

Majewski, S. R., Wilson, J. C., Hearty, F., Schiavon, R. R., \& Skrutskie, M. E. 2010, in IAU Symp. 265, Chemical Abundances in the Universe: Connecting First Stars to Planets, ed. K. Cunha, M. Spite, \& B. Barbuy (Cambridge: Cambridge Univ. Press), 480

Majewski, S. R., Zasowski, G., \& Nidever, D. L. 2011, ApJ, 739, 25

Marigo, P., Girardi, L., Bressan, A., et al. 2008, A\&A, 482, 883

Mathur, S., García, R. A., Régulo, C., et al. 2010, A\&A, 511, 46

Matteucci, F., Spitoni, E., Recchi, S., \& Valiante, R. 2009, A\&A, 501, 531

McQuillan, A., Mazeh, T., \& Aigrain, S. 2014, ApJS, 211, 24 
Meszaros, S., Holtzman, J., García Pérez, A. E., et al. 2013, AJ, 146, 133 Miglio, A., Brogaard, K., Stello, D., et al. 2012, MNRAS, 419, 2077 Miglio, A., Chiappini, C., Morel, T., et al. 2013, MNRAS, 429, 423

Miglio, A., Montalbán, J., Eggenberger, P., et al. 2009, A\&A, 503, L21

Mosser, B., Barban, C., Montalbán, J., et al. 2011a, A\&A, 532, 86

Mosser, B., Belkacem, K., Goupil, M. J., et al. 2010, A\&A, 517, 22

Mosser, B., Belkacem, K., Goupil, M. J., et al. 2011b, A\&A, 525, L9

Mosser, B., Goupil, M. J., Belkacem, K., et al. 2012a, A\&A, 548, 10

Mosser, B., Goupil, M. J., Belkacem, K., et al. 2012b, A\&A, 540, 143

Perryman, M. A. C., de Boer, K. S., Gilmore, G., et al. 2001, A\&A, 369, 339

Pietrinferni, A., Cassisi, S., Salaris, M., \& Castelli, F. 2004, ApJ, 612, 168

Pinsonneault, M. H., An, D., Molenda-Żakowicz, J., et al. 2012, ApJS, 199, 30

Rodrigues, T. S., Girardi, L., Miglio, A., et al. 2014, MNRAS, 445, 2758

Serenelli, A., Bergemann, M., Ruchti, G., \& Casagrande, L. 2013, MNRAS, 429,3645

Silva Aguirre, V., Basu, S., Brandão, I. M., et al. 2013, ApJ, 769, 141

Silva Aguirre, V., Casagrande, L., Basu, S., et al. 2012, ApJ, 757, 99

Silva Aguirre, V., Chaplin, W. J., Ballot, J., et al. 2011, ApJL, 740, L2

Skrutskie, M. F., Cutri, R. M., Stiening, R., et al. 2006, AJ, 131, 1163
Smith, J. C., Stumpe, M. C., van Cleve, J. E., et al. 2012, PASP, 124, 1000

Stello, D., Chaplin, W. J., Basu, S., Elsworth, Y., \& Bedding, T. R. 2009a, MNRAS, 400, L80

Stello, D., Chaplin, W. J., Bruntt, H., et al. 2009b, ApJ, 700, 1589

Stello, D., Huber, D., Bedding, T. R., et al. 2013, ApJL, 765, L41

Stello, D., Huber, D., Kallinger, T., et al. 2011a, ApJL, 737, L10

Stello, D., Meibom, S., Gilliland, R. L., et al. 2011b, ApJ, 739, 13

Stumpe, M. C., Smith, J. C., van Cleve, J. E., et al. 2012, PASP, 124,985

Tassoul, M. 1980, ApJS, 43, 469

Thygesen, A. O., Frandsen, S., Bruntt, H., et al. 2012, A\&A, 543, 160

Tinsley, B. 1979, ApJ, 229, 1046

Ulrich, R. K. 1986, ApJL, 306, L37

Wallerstein, G. 1962, ApJS, 6, 407

Weiss, A., \& Schallt, H. 2008, Ap\&SS, 316, 99

White, T. R., Bedding, T. R., Stello, D., et al. 2011, ApJ, 743, 161

Yanny, B., Rockosi, C., Newberg, H. J., et al. 2009, AJ, 137, 4377

Zasowski, G., Johnson, J. A., Frinchaboy, P. M., et al. 2013, AJ, 146, 81

Zhao, G., Chen, Y.-Q., Shi, J.-R., et al. 2006, ChJAA, 6, 265 